\title{
Comparison of accelerated ion populations observed upstream of the bow shocks at Venus and Mars
}

\author{
M. Yamauchi ${ }^{1}$, Y. Futaana ${ }^{1}$, A. Fedorov ${ }^{2}$, R. A. Frahm ${ }^{3}$, J. D. Winningham ${ }^{3}$, E. Dubinin ${ }^{4}$, R. Lundin ${ }^{1}$, S. Barabash ${ }^{1}$, \\ M. Holmström ${ }^{1}$, C. Mazelle $^{2}$, J.-A. Sauvaud ${ }^{2}$, T. L. Zhang ${ }^{5}$, W. Baumjohann ${ }^{5}$, A. J. Coates ${ }^{6}$, and M. Fraenz ${ }^{4}$ \\ ${ }^{1}$ Swedish Institute of Space Physics, Box 812, 98128 Kiruna, Sweden \\ ${ }^{2}$ Centre d'Etude Spatiale des Rayonnements, Université de Toulouse, 31038 Toulouse, France \\ ${ }^{3}$ Southwest Research Institute, San Antonio, TX 78228-0510, USA \\ ${ }^{4}$ Max-Planck-Institut für Sonnensystemforschung, 37191 Katlenburg-Lindau, Germany \\ ${ }^{5}$ Space Research Institute, Austrian Academy of Sciences, 8042 Graz, Austria \\ ${ }^{6}$ Mullard Space Science Laboratory, University College London, Surrey, RH5 6NT, UK
}

Received: 29 March 2010 - Revised: 3 February 2011 - Accepted: 27 February 2011 - Published: 10 March 2011

\begin{abstract}
Foreshock ions are compared between Venus and Mars at energies of $0.6 \sim 20 \mathrm{keV}$ using the same ion instrument, the Ion Mass Analyser, on board both Venus Express and Mars Express. Venus Express often observes accelerated protons $(2 \sim 6$ times the solar wind energy) that travel away from the Venus bow shock when the spacecraft location is magnetically connected to the bow shock. The observed ions have a large field-aligned velocity compared to the perpendicular velocity in the solar wind frame, and are similar to the field-aligned beams and intermediate gyrating component of the foreshock ions in the terrestrial upstream region. Mars Express does not observe similar foreshock ions as does Venus Express, indicating that the Martian foreshock does not possess the intermediate gyrating component in the upstream region on the dayside of the planet. Instead, two types of gyrating protons in the solar wind frame are observed very close to the Martian quasi-perpendicular bow shock within a proton gyroradius distance. The first type is observed only within the region which is about $400 \mathrm{~km}$ from the bow shock and flows tailward nearly along the bow shock with a similar velocity as the solar wind. The second type is observed up to about $700 \mathrm{~km}$ from the bow shock and has a bundled structure in the energy domain. A traversal on 12 July 2005, in which the energy-bunching came from bundling in the magnetic field direction, is further examined. The observed velocities of the latter population are consistent with multiple specular reflections of the solar wind at the bow shock, and the ions after the second reflection have a fieldaligned velocity larger than that of the de Hoffman-Teller velocity frame, i.e., their guiding center has moved toward in-
\end{abstract}

Correspondence to: $\mathrm{M}$. Yamauchi (m.yamauchi@irf.se) terplanetary space out from the bow shock. To account for the observed peculiarity of the Martian upstream region, finite gyroradius effects of the solar wind protons compared to the radius of the bow shock curvature and effects of cold ion abundance in the bow shock are discussed.

Keywords. Interplanetary physics (Energetic particles; Planetary bow shocks) - Space plasma physics (Charged particle motion and acceleration)

\section{Introduction}

Upstream of the terrestrial bow shock, back-streaming energetic protons are often found flowing nearly along the interplanetary magnetic field (IMF) away from the bow shock when the location is magnetically connected to the bow shock (Asbridge et al., 1968; Gosling et al., 1978; Paschmann et al., 1981; Sckopke et al., 1983; Fuselier et al., 1986; Meziane et al., 2004b; for review, Eastwood et al., 2005; Bale et al., 2005). This is the foreshock region. These energetic ion populations have been subdivided into three categories according to their distribution functions: ions flowing nearly along the IMF in the solar wind frame within a limited energy range about $2 \sim 6$ times that of the solar wind, nearly isotropic ions in a wide energy range, and ions possessing the same energy range as the first category but flowing with finite angle to the IMF in the solar wind frame. The last category is further subdivided depending on its probable seeding population, i.e., one that is most likely reflected solar wind at the bow shock, and one that is most likely a partially isotropized form of the first category. Which of four categories is observed depends on the observer's location with respect to both the bow shock and the IMF.

Published by Copernicus Publications on behalf of the European Geosciences Union. 


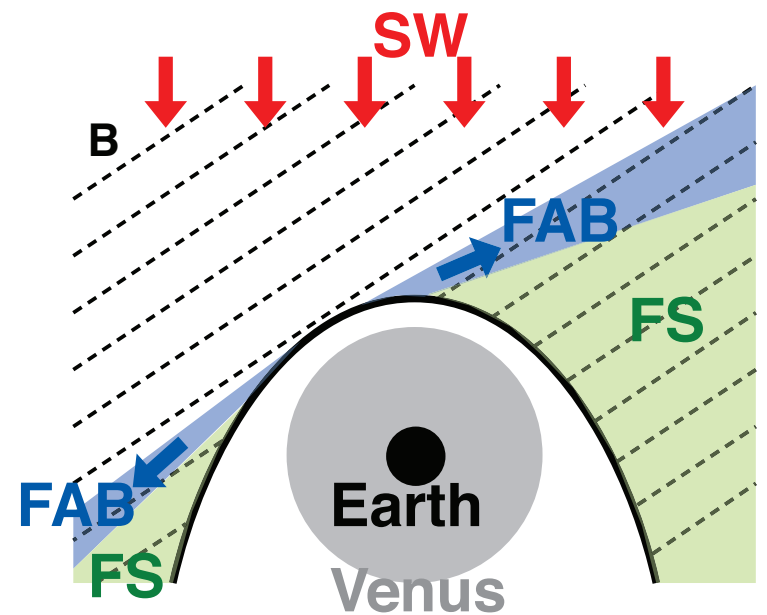

Fig. 1. Schematic illustration of the foreshock (FS) location. Fieldaligned ions (FAB) are often observed at the upstream boundary of the foreshock region. Relative sizes of the planets compared to the bow shock are also drawn.

The first category is called the "field-aligned beams," and is observed at the upstream boundary of the foreshock region, i.e., where the magnetically-connected bow shock is a quasiperpendicular shock as illustrated in Fig. 1 with blue hatch (Gosling et al., 1978). The second category is called the "diffuse component," and is observed when the observer's location is connected to quasi-parallel shocks as marked with green hatch in Fig. 1 (Gosling et al., 1978). The last two categories are called the "reflected-gyrating component" when it is observed close to quasi-perpendicular shocks (Sckopke et al., 1983) and the "intermediate (gyrating) component" when it is observed downstream of the region where field-aligned beams are found (Fuselier et al., 1986). Both the reflectedgyrating component and the intermediate (gyrating) component can be either gyrotropic or gyrophase-bunched (Sckopke et al., 1983; Fuselier et al., 1986; Möbius et al., 2001), but this information is not used for categorization.

Since the formation mechanisms of the field-aligned beams and the intermediate component are considered to be similar to each other (Kucharek et al., 2004), the intermediate component is sometimes categorized as field-aligned beams. However, we do not use such a combined naming in this paper. Instead, we call the last two categories as the "gyrating component" based on the distribution function because this paper deals with foreshocks of Mars and Venus, where the formation mechanisms of the foreshock ions can be different from those at the Earth.

Both the field-aligned beams and the intermediate component have lower alpha/proton ratios than that in the original solar wind or in the diffuse component (Fuselier and Thomsen, 1992), and their sources are considered to be either the reflected solar wind (e.g., Gosling et al., 1978; Paschmann et al., 1980; Mazelle et al., 2003; Kucharek et al., 2004;
Meziane et al., 2004a), or heated magnetosheath ions that leak through the bow shock along the magnetic field (Tanaka et al., 1983). The leakage scenario involves ion heating by electromagnetic waves downstream of the shock (Tanaka et al., 1983), and the reflection scenario normally employs a simplified assumption of specular reflection of the solar wind by a quasi-perpendicular shock (Paschmann et al., 1980). Another type of leakage scenario from deep inside the magnetosphere is proposed by Sarris et al. (1987), in which the relevant energy is much higher than that of these two populations.

The microphysics of the specular reflection is an open question. We do not yet know how only a part of the solar wind is reflected into a very specific direction, whereas a majority of the solar wind flows toward the magnetosheath on the downstream side of the bow shock. With a specular reflection that conserves the ion speed in the planetary frame, the reflected solar wind protons can have up to twice the solar wind speed $\left(V_{\mathrm{SW}}\right)$ in the solar wind frame, and up to $3 V_{\mathrm{SW}}$ (nine times the solar wind proton energy) in the planetary frame after $180^{\circ}$ of gyration, i.e., when its gyrophase points toward the anti-sunward direction (Paschmann et al., 1980, 1981). Such energetic ions are actually observed in the upstream region of the Moon, whose surface reflects a significant amount of the solar wind (Nishino et al., 2009; Holmström et al., 2010).

The conversion of the energy from the perpendicular direction to the parallel direction with respect to the magnetic field can be either by pitch-angle scattering or multiple bouncing in the foot region immediately after reflection (Giacalone et al., 1994; Möbius et al., 2001; Oka et al., 2005). The reflection scenario also explains the low alpha/proton ratio because an alpha particle has a larger inertia (mass per charge) than a proton if the velocity is the same. Once the ions have escaped from the bow shock, their field-aligned velocities do not change as long as the IMF is uniform because the Lorentz force does not act in the field-aligned direction. Depending on the ratio of the beam's field-aligned velocity $\left(V_{\|}\right)$and the solar wind velocity component perpendicular to the magnetic field $\left(V_{\mathrm{SW}} \perp\right)$, these ions experience a so-called velocity filter effect to either completely escape from the bow shock within the magnetically-connected foreshock region or return back to the bow shock (Eastwood et al., 2005; Sibeck et al., 2008). To diagnose whether the ions will escape or return, it is useful to obtain the field-aligned velocity in the de Hoffman-Teller frame (Miao et al., 2009, and references therein).

The average electric potential drop across the bow shock is a few hundred volts under an assumption of zero fieldaligned electric field (Eastwood et al., 2007, and references therein), and is formed as a result of the proton inertia. Proton motions that form the foreshock also require the consideration of the proton inertia. In a magnetized plasma, there are two scale sizes relevant to the proton inertia: the ion gyroradius (without polarization electric field) and the ion inertia length (with polarization electric field). Therefore, the radius 
Table 1. Parameters of Earth and Mars as compared to Venus.

\begin{tabular}{lccccc}
\hline planet & $V_{\mathrm{SW}}$ & $M_{\mathrm{A}} \propto n_{\mathrm{SW}}^{1 / 2} V_{\mathrm{SW}} / B$ & $\mathrm{c} / \omega_{\mathrm{pi}} \propto n_{\mathrm{SW}}^{-1 / 2}$ & $r_{\mathrm{g}} \propto V_{\mathrm{SW}} / B$ & bow shock size \\
\hline Venus & 1 & 1 & 1 & 1 & 1 \\
Earth & $\sim 1$ & $\sim 1.2$ & $\sim 1.4$ & $\sim 1.7$ & $>5$ \\
Mars & $\sim 1$ & $\sim 1.4$ & $\sim 2$ & $\sim 3$ & $\sim 0.5$ \\
\hline
\end{tabular}

$V_{\mathrm{SW}}:$ solar wind speed (Smith and Wolfe, 1979)

$M_{\mathrm{A}}$ : Alfvén Mach number (Savin et al., 1984)

$n_{\mathrm{SW}}$ : solar wind density

$B$ : interplanetary magnetic field strength

$c / \omega_{p i}$ : proton inertia length $\left(\sim 100 \mathrm{~km}\right.$ for $\left.5 \mathrm{~cm}^{-3} \mathrm{H}^{+}\right)$

$r_{g}$ : proton gyroradius $\left(\sim 1000 \mathrm{~km}\right.$ for $2 \mathrm{keV} \mathrm{H}^{+}$under $\left.6 \mathrm{nT} \mathrm{B}\right)$

of the bow shock curvature near the subsolar point (hereafter referred to as "bow shock size" in this paper) compared to the proton gyroradius (about $1000 \mathrm{~km}$ for the solar wind proton) and to the proton inertia length (order of $100 \mathrm{~km}$ in the foot region) can be key parameters that determine the characteristics (energy, intensity, and distribution) of the foreshock ions. From this viewpoint, it is meaningful to compare the foreshocks of the Earth, Venus and Mars because the bow shock size is more than $50000 \mathrm{~km}$ for the Earth, $\sim 10000 \mathrm{~km}$ for Venus, and only $\sim 5000 \mathrm{~km}$ for Mars, whereas the gyroradius of a $4 \mathrm{keV}$ proton in a $4 \mathrm{nT}$ magnetic field is about $2000 \mathrm{~km}$. The bow shock curvature can no longer be ignored when considering the motion of reflected (accelerated) ions at Mars, but it can be ignored for the Earth or Venus.

A rough comparison of the solar wind plasma parameters (their ratios) among the regions near Venus, the Earth, and Mars is listed in Table 1, in which we adopt the Alfvén Mach number $\left(M_{\mathrm{A}}\right)$ from Slavin et al. (1984) and a constant solar wind speed between Venus and Mars (Smith and Wolfe, 1979). The Venus-Earth difference in the bow shock size is due to the intrinsic dipole magnetic field that exists only at the Earth. The Venus-Mars difference in the bow shock size is due to the difference in the planetary size. According to Table 1, the ratio of gyroradius to bow shock size increases by a factor of more than 3 from the Earth to Venus, and by a factor of about $5 \sim 6$ from Venus to Mars. Similar differences between the planets are also seen in the ratio of proton inertia length to bow shock size, but the proton inertia length at Mars (few hundred $\mathrm{km}$ ) is still very small compared to the Martian bow shock size $(\sim 5000 \mathrm{~km})$. The Mach numbers (Alfvén and Fast Magnetosonic) are similar among these three planets, varying only by a factor of $\sim 1.4$ between Venus and Mars (Slavin et al., 1984).

There is another important difference among Mars, Venus, and the Earth: the abundance of cold ions in the bow shock (Dubinin et al., 1993). The bow shock is within the hydrogen exosphere of Mars but far beyond the exosphere of the Earth. In fact, pick-up ions of newly ionized hydrogen from the exosphere are regularly observed upstream of the Martian bow shock (Barabash et al., 1991; Dubinin et al., 1995,
2006; Yamauchi et al., 2006), which is not the case for the Earth. This difference comes from the relative locations between the bow shocks and the exosphere (the source of cold ions). The Venus case is unclear, but at least the amount of cold ions in bow shock at Venus is much less than at Mars.

The Martian bow shock can trap these cold ions into the internal electric potential structure (e.g., Grard et al., 1991), resulting in a substantial difference in the bow shock dissipation mechanism between the Earth and Mars. Such cold ions inside the Martian bow shock have been observed by Phobos2 spacecraft (Dubinin et al., 1993). Dubinin et al. showed a sudden appearance of low-energy protons at the bow shock and estimated the number density of the exospheric-origin ions, which turned out to be comparable with the number density of shocked solar wind protons.

Past foreshock investigations of Venus and Mars have been conducted mostly by using electron, magnetic field, and wave observations (e.g., Mazelle et al., 2004, and references therein). Ion observations in the foreshock region near these planets were limited to Phobos-2 observations at Mars (Barabash and Lundin, 1993; Dubinin et al., 1995, 2000). The present knowledge is insufficient to understand the difference between the ion populations in the foreshock regions of the different planets. Mars Express (MEX) and Venus Express (VEX) are ideal missions to compare the foreshock between Mars and Venus because both missions carry the identical instruments that cover the energy range of foreshock plasma. The Ion Mass Analyser (IMA) and ELectron Spectrometer (ELS) are parts of the Analyzer of Space Plasma and Energetic Atoms (ASPERA-3 for MEX and ASPERA-4 for VEX) plasma packages. Using IMA data, we show case studies that demonstrate the difference and similarity of the foreshock ions between these planets.

\section{Data}

The IMA is a top hat instrument that combines an electrostatic energy analyzer with a magnetic mass analyzer. IMA measures ions in the energy range from $10 \mathrm{eV}$ per charge to 


\section{Azimuth $(\phi)$ direction}

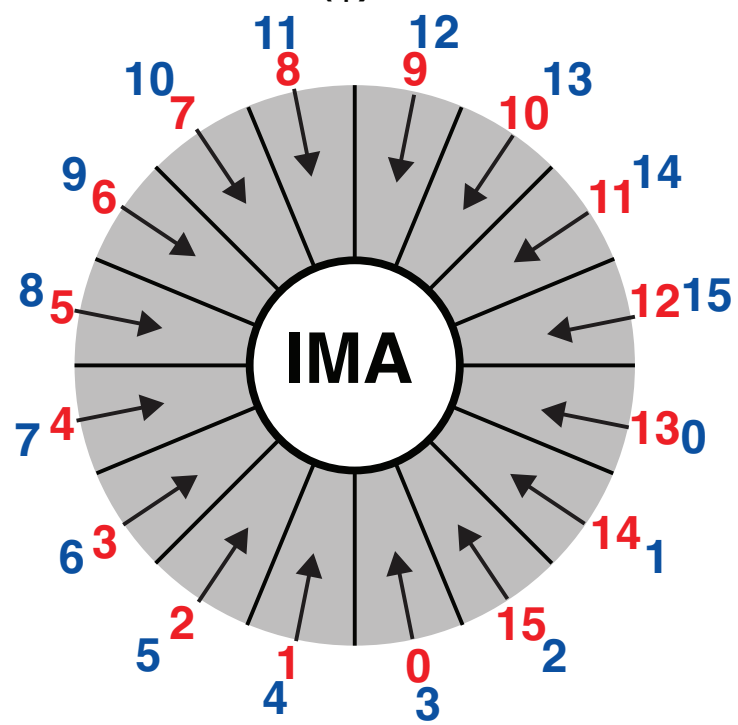

blue: VEX FOV red: MEX FOV

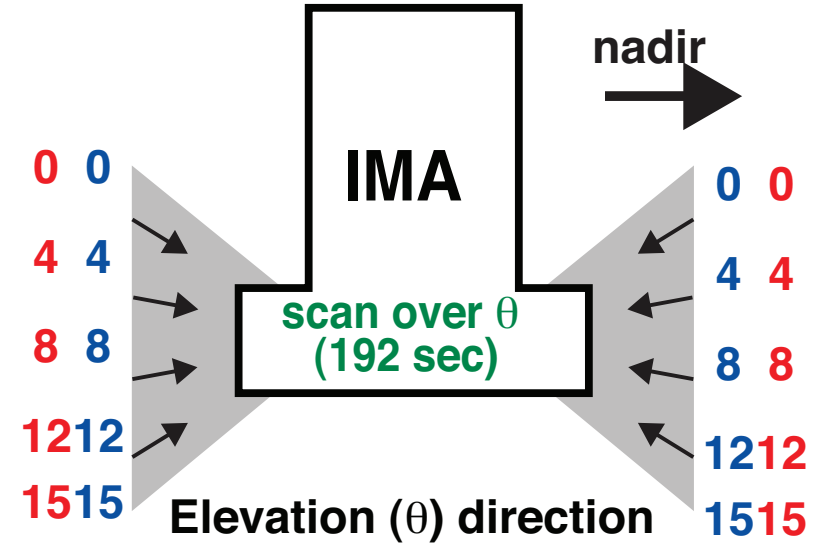

Fig. 2. Definition of field-of-view directions $(\theta$ and $\phi)$ of IMA for both Venus Express (blue) Mars Express (red). The azimuthal sector numbers $(\phi)$ are given in the upper panel, and the elevation numbers $(\theta)$ are given in the lower panel.

$30 \mathrm{keV}$ per charge in 96 logarithmically spaced energy steps every $12 \mathrm{~s}$. IMA has a $4.6^{\circ} \times 360^{\circ}$ field of view, where the $360^{\circ}$ measurement plane is divided into 16 azimuthal sectors $(\phi=0-15)$, each $22.5^{\circ}$ wide. IMA has an extra electrostatic deflection system (or elevation analyzer) at its entrance, which scans 16 directions from $-45^{\circ}$ to $+45^{\circ}(\theta=0$ $15)$ in $192 \mathrm{~s}$. Thus, the overall field-of-view is approximately $360^{\circ}(16$ sectors in $\phi) \times 90^{\circ}(16$ elevations in $\theta)$. Both azimuthal sectors ( $\phi$ direction) and elevations ( $\theta$ direction) are numbered as shown in Fig. 2. The definition of the sector numbers with respect to the nadir direction is different between VEX and MEX. For details of the IMA instrument, see Barabash et al. (2006), Fedorov et al. (2006), and Yamauchi et al. (2006).
In order to examine the distribution of the energetic ions in the foreshock region, it is necessary to know the orientation of the IMF. Unfortunately, MEX does not carry a magnetometer, and hence the IMF orientation must be estimated from the ring distribution of newly-born ions coming from the planetary exosphere when these "primary" ring ions are observed within the field-of-view of IMA and when the IMA operation mode (i.e., post acceleration setting) is optimum for the measurement of light ions (Yamauchi et al., 2006, 2008). The present analysis at Mars has an extra difficulty because separating the primary ring distribution from the foreshock ions is not a simple task without the measurement of the magnetic field. In such convoluted populations, one may not obtain a reliable IMF orientation from the IMA data unless the IMF is stable for more than a few elevation scans, i.e., more than ten minutes. After examining quicklook spectrograms (integrated over all 16 azimuth directions) during 2004 and 2005, we have identified several traversals with multiple energy structures (primary ring distribution and accelerated ions are clearly separated in the energy domain) that are stable for more than several scans.

\section{Venus observation}

MEX and VEX data were examined for signatures from the foreshock field-aligned beams and gyrating component during time periods when IMA performed full elevation $(\theta)$ scan (16 directions). Unfortunately, IMA on VEX performed full elevation scanning only during the first month of operation (14 May 24 June 2006). Yet, VEX/IMA observed the foreshock ion signatures in more than 10 traversals (out of about 30 traversals) in the upstream region of Venus. Among those, the data from 18 June 2006 was chosen because the IMF changed in step-like fashion between two directions: the VEX location and the bow shock are magnetically connected during one period and disconnected before and after. Figures 3-5 show the data from this traversal.

Figure 3 shows the orbit (two top panels), magnetic field (second panel), data of two ELS sectors (third and fourth panels that correspond to two opposite viewing directions), and proton data from the IMA (bottom panel) on 18 June 2006 during 00:00 01:50 UT. The Venus-Sun Orbit (VSO) Cartesian coordinate system is used; i.e., the $+\boldsymbol{X}$ direction points sunward, the $+\boldsymbol{Z}$ direction is perpendicular to the planet's orbital plane and points northward, the $+\boldsymbol{Y}$ direction completes the right-handed Cartesian coordinate system. IMA data are integrated over all 16 azimuthal sctors $(\phi)$.

Figure 4 shows the IMA proton data for azimuthal sectors $\phi=3 \sim 8$ separated into each $192 \mathrm{~s}$ scan during 00:34:23 00:59:59 UT (8 scans). Each window (elevation scan) in Fig. 4 shows an elevation-energy spectrogram ranging from $-45^{\circ}$ to $+45^{\circ}(\theta=0-15)$ at a specific azimuth $(\phi)$ every $192 \mathrm{~s}$. The definitions of $\theta$ and $\phi$ are given in Fig. 2. 


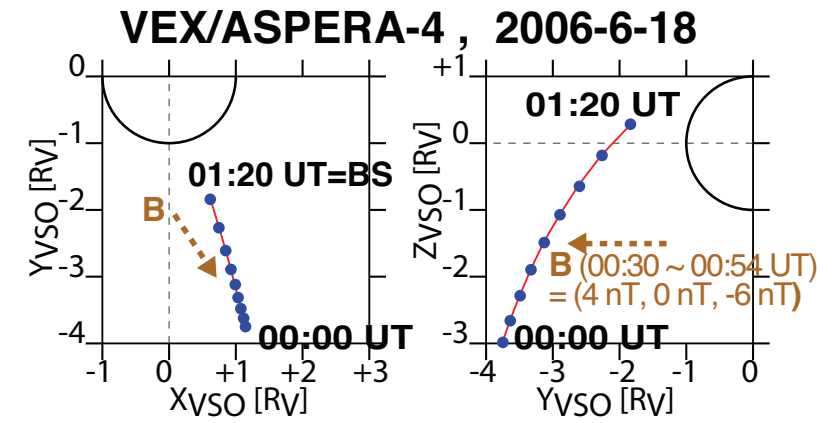

MAG \& ELS \& IMA data, 00:00 01:40 UT

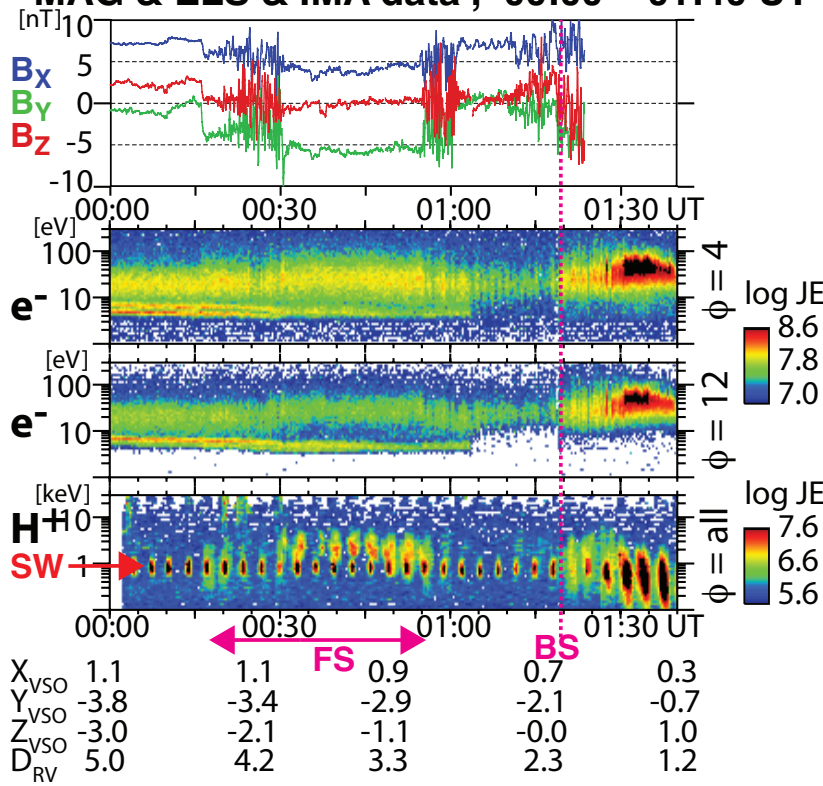

Fig. 3. Overview of the VEX orbit, magnetic field, and hot plasma data (bottom three panels) on 18 June 2006 during 00:00 01:40 UT. The two top panels show the VEX orbit in the VSO coordinate system (see text for definition). The VEX traversal is drawn by a red line with ticks every $10 \mathrm{~min}$. The unit $R_{\mathrm{V}}$ is the Venus radius. The second panel shows the magnetic field $X$ (blue), $Y$ (green), and $Z$ (red) components in the VSO coordinate. The bottom three panels show energy-time spectrograms of the energy flux ( $\mathrm{keV} \mathrm{cm}^{-2} \mathrm{~s}^{-1} \mathrm{keV}^{-1}$ ) of electrons (two opposite directions) and protons (average of all 16 azimuthal directions). The nearly 3-min (192 s) cycle seen in the IMA data is due to the scanning cycle of the IMA entrance (elevation) direction from $\theta=0$ to $\theta=15$. The solar wind protons (SW) are seen at around $1 \mathrm{keV}$ every $192 \mathrm{~s}$ scanning cycle. The bow shock (BS) is crossed at around 01:20 UT, and foreshock ions (FS) are observed at around 00:15 01:00 UT.

Figure 5 shows the velocity space distribution function against $V_{\perp}$ (horizontal axis) and $V_{\|}$(horizontal axis) in the spacecraft frame during these 8 scans, where $\perp$ and $\|$ denote perpendicular and parallel directions with respect to the magnetic field, respectively. The perpendicular direction in this figure is defined so that the $V_{\perp}-V_{\|}$plane shown in each panel contains both the magnetic field direction and the solar wind flow direction. Therefore, the vertical red dashed line corresponds to $V_{\perp}=0$ in the solar wind frame. Note that IMA does not cover $4 \pi$ steradian, and some of the viewing directions, e.g., most of $\phi=12 \sim 15$ and half $(\theta=0 \sim 6)$ of $\phi=0 \sim 2$, are blocked by the spacecraft.

In Fig. 3, an increase in the proton flux with energies $1 \sim 6 \mathrm{keV}$ is observed during 00:16 00:57 UT (marked by FS). This flux is intensified during 00:31 00:55 UT. Only during this period, the Y-component of the average IMF $\left(B_{Y}\right)$ is larger than the other components, i.e., the spacecraft location is magnetically connected to the bow shock. The ELS data also show that $10 \sim 50 \mathrm{eV}$ electrons are intensified during the same time period when the proton flux increases. They fluctuate in both intensity and energy when the IMF also fluctuates at the beginning and end of this foreshock interval, which indicates the existence of waves that propagate along the IMF from the bow shock. Such structured electrons normally indicate a magnetic connection to the bow shock.

The $1 \sim 6 \mathrm{keV}$ intense protons during this period are observed in a limited azimuthal direction $(\theta=4 \sim 7)$ as shown in Fig. 4. These non-solar wind ions have high $\left|V_{\|}\right|$according to Fig. 5, and are traveling away from the bow shock along the magnetic field. These ions have a perpendicular velocity $V_{\perp}=0 \sim 2 V_{\mathrm{SW} \perp}$ in the spacecraft frame; i.e., the perpendicular speed in the solar wind frame is about $\leq V_{\mathrm{SW} \perp}$. Thus, this population has a gyrating distribution with a large $\left|V_{\|}\right|$ (including field-aligned beam), in the same manner as the field-aligned beams and gyrating component of the terrestrial foreshock ions.

Considering the magnetic field polarity, the Lorentz force on an ion that is registered at $\theta>9$ bends the ion flow direction from the $\phi=7$ direction to the $\phi=4$ direction during its gyration. Similarly, an ion that is registered at $\theta<6$ is expected to be bent from the $\phi=4$ direction to the $\phi=7$ direction. Unfortunately, this information does not help in judging whether the gyrating component is gyrotropic or gyrophase bunched, because the IMA look direction is limited by substantial blockage due to the spacecraft, and because the observation cycle ( $12 \mathrm{~s}$ for one elevation $(\theta)$ direction) is rather slow (cf. proton gyroperiod is about $4 \mathrm{~s}$ ) such that the ion distribution can change before completing a full angle scan in $192 \mathrm{~s}$. In other words, some of the variations in the elevation $(\theta)$ direction must be interpreted as temporal variations rather than spatial changes. For example, in the first windows (00:34:23 00:37:35 UT) of Fig. 4, a gyrotropic distribution predicts ion counts at $\theta<13$ for $\phi=4$, but they are not observed. The absence of ion flux in the expected direction can well be due to a temporal variation.

With these limitations in mind, the energy range $(1 \sim 6$ times the solar wind energy) and flow direction (high $\left|V_{\|}\right|$ with limited $\left.\left|V_{\perp}\right|\right)$ of the observed back-streaming ions in the Venus foreshock are similar to those in the terrestrial foreshock (field-aligned beams and gyrating component). Thus, Venus has similar foreshock ion structures as those at the Earth despite the different standoff distances of the bow shocks between Venus and the Earth. The fact that all 


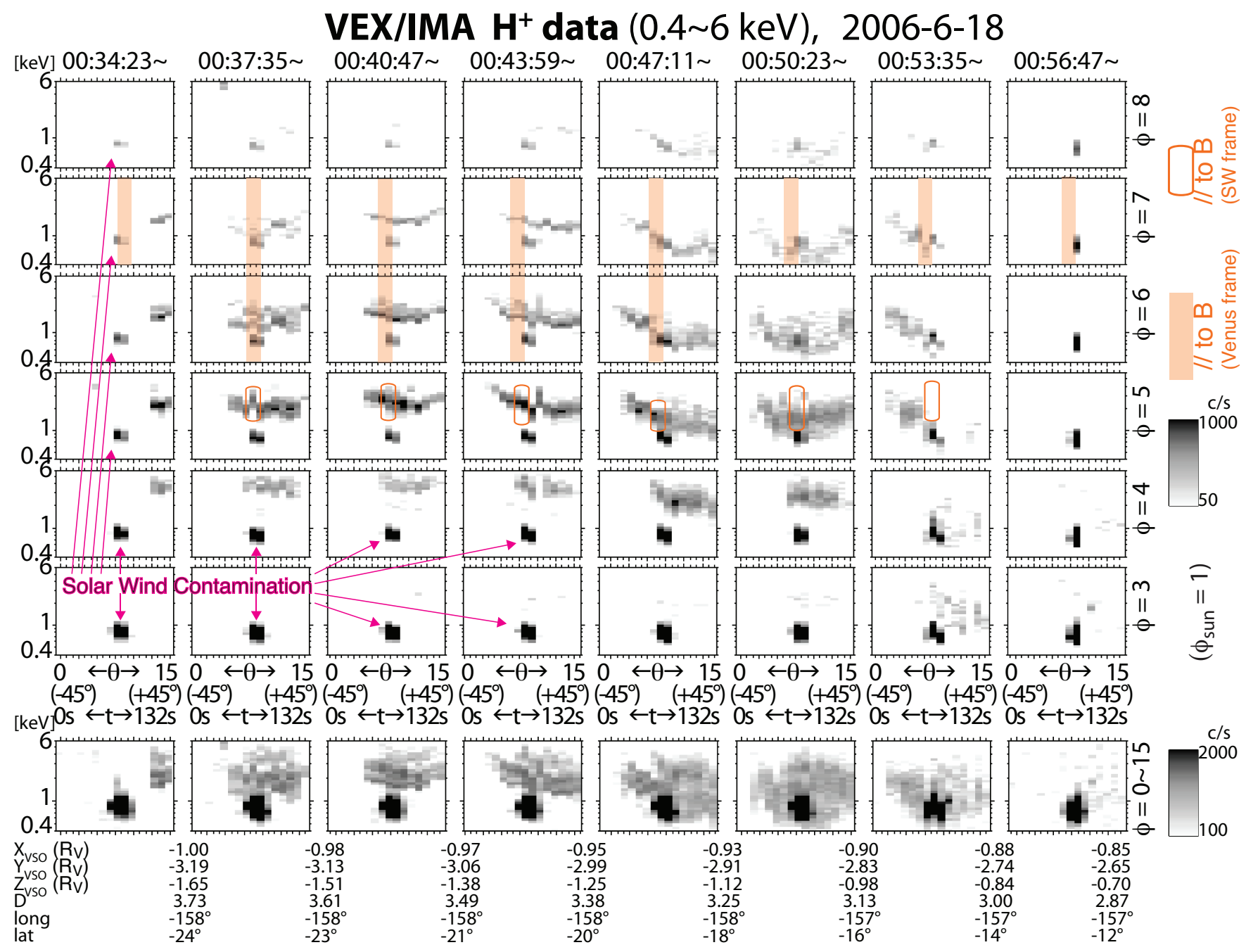

Fig. 4. Energy-angle spectrograms from VEX/IMA proton channels $(0.4 \sim 6 \mathrm{keV})$ on 18 June 2006 as they progress over time during 00:34:23 00:59:59 UT (part of Fig. 3). The ion species (proton) is confirmed by the energy-mass matrix (not shown here). Six different azimuthal sectors (from top to bottom $\phi=8,7,6,5,4,3$ ) are shown for each $192 \mathrm{~s}$ scanning cycle in elevation, such that each window corresponds to an elevation-energy spectrogram for elevation angles $-45^{\circ} \sim+45^{\circ}(\theta=0-15)$. The magnetic field direction in the spacecraft frame is indicated by filled red squares. Although the magnetic field direction is about the same between the spacecraft frame and the solar wind frame, the field-aligned direction for ions in the solar wind frame is shifted by the solar wind velocity (cf. Fig. 5, marked by empty orange squares). The energy dependency of this direction $(\theta$ and $\phi)$ is very weak for the foreshock ions that we discuss here, because the field-aligned velocity of these ions is very large.

foreshock ions have a perpendicular speed of about $\leq V_{\mathrm{SW} \perp}$ in the solar wind frame, suggests that the gyrating component originates from $V_{\perp}=0$ in the planetary frame, similar to the way pick-up ions are generated, except that the observed foreshock ions have large $\left|V_{\|}\right|$.

Both the field-aligned beams and gyrating components of the Venus foreshock ions have a large $\left|V_{\|}\right|$with a small width $\left(\delta V_{\|}\right)$, forming beam-like distributions. The small $\delta V_{\|}$suggests field-aligned potential acceleration, specular reflection of the solar wind beam, or significant velocity filtering due to long time-of-flight. Since the distance from VEX to the bow shock is about $10000 \mathrm{~km}$ at approximately 00:50 UT (Fig. 3) and the field-aligned velocity is about $300 \sim 500 \mathrm{~km} \mathrm{~s}^{-1}$ (Fig. 5), the travel time is only $20 \sim 30 \mathrm{~s}$ for the observed foreshock protons and about $50 \mathrm{~s}$ for $200 \mathrm{~km} \mathrm{~s}^{-1}(0.2 \mathrm{keV})$ protons, the latter of which are not observed. The difference in the time-of-flight between these ions is small enough for VEX to simultaneously detect protons with both energies if the source population at the bow shock had a broad energy spectrum. Therefore, the small $\delta V_{\|}$cannot be attributed to a velocity filter effect, in agreement with recent studies at the terrestrial foreshock (e.g., Meziane et al., 2004b; Oka et al., 2005). 


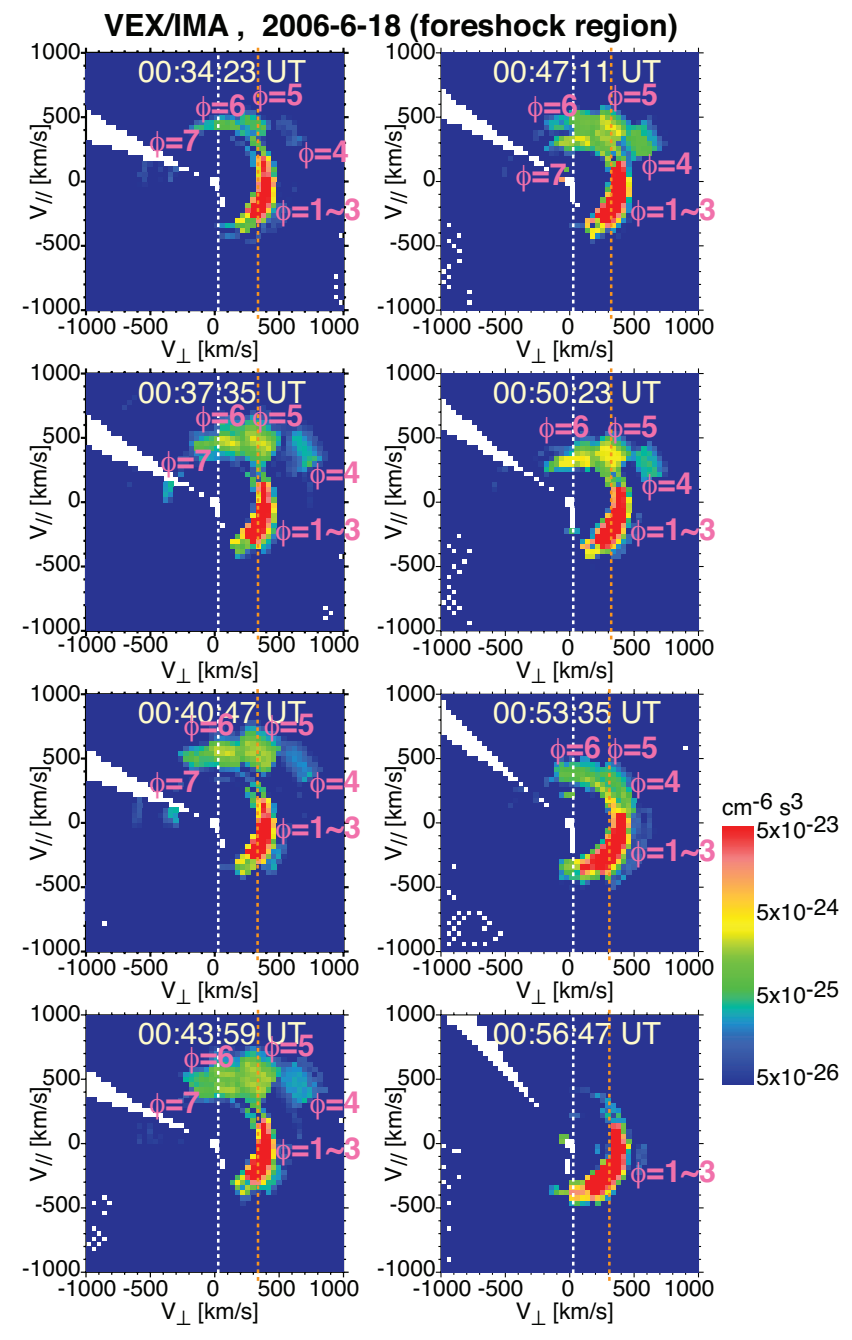

Fig. 5. Velocity-space distribution function $\left(V_{\perp}\right.$ and $V_{\|}$axes, where $\perp$ and $\|$ denote perpendicular and parallel directions with respect to the magnetic field) for the eight scans (192 s cycle for each scan) shown in Fig. 4 (00:34:23 00:59:59 UT). Each panel shows a 2-D projection of the 3-D distribution function, where this 2-D plane is defined by the magnetic field direction and the solar wind flow direction. Therefore, the dashed vertical red lines denote the perpendicular velocity of the solar wind $\left(V_{\mathrm{SW}}\right)$, and this is the $V_{\|}$axis in the solar wind reference frame. The corresponding IMA look directions are $\theta=7 \sim 9$ at $\phi=5$ and $\theta=7 \sim 8$ at $\phi=1$ (marked by empty orange squares in Fig. 4). The dashed vertical white lines denote $V_{\perp}=0$ in the spacecraft frame, and the corresponding IMA look directions are $\theta=6 \sim 8$ at $\phi=6 \sim 7$ and $\theta=7 \sim 9$ at $\phi=14 \sim 15$ (marked by filled red squares in Fig. 4). Its opposite phase point in the solar wind reference frame corresponds to $\phi=4$. The separation of the peaks between neighboring azimuthal sectors (between $\phi=4, \phi=5$, and $\phi=6$ ) is most likely real because the registered energy in Fig. 4 is quite different between these sectors.

Therefore, the observed small $\delta V_{\|}$compared to $\left|V_{\|}\right|$indicates that the source population comes either from cold ions (field-aligned potential acceleration scenario) or the so- lar wind (specular reflection scenario), but cannot be heated magnetosheath ions. The cold ion scenario is possible for non-magnetized planets with small bow shock standoff distance compared to the Earth because cold ions can be abundant at the bow shock (Dubinin et al., 1993). However, at Venus, this situation is not as clear as at Mars.

\section{Mars observation}

The entire MEX/IMA set of data during the first two years (2004-2005) when MEX was inside the solar wind region was surveyed for similar ion distributions as those shown in Fig. 3. Although MEX's apoaxis is much lower than VEX's apoaxis, MEX/IMA made measurements in the upstream region up to $3000 \mathrm{~km}$ from the bow shock in the solar wind on the dayside before the terminator. In about 500 traversals through the upstream region, no traversal with detectable accelerated ions similar to the data as shown in Figs. 3-5 was found. Based on the probability of the IMF orientation at Mars, we may expect at least 50 foreshock traversals if Mars has a similar foreshock to those at the Earth or Venus. The lack of similar ion distributions as those observed in the Venus foreshock region using the identical instrument means that at least one of two populations (field-aligned beams or gyrating component) is absent. The MEX/IMA cannot miss detecting the gyrating component because it occupies a large elevation $(\theta)$ and azimuth $(\phi)$ as shown in Fig. 4, whereas it is difficult without magnetometer to judge whether a fieldaligned beam exists or is absent when the accompanying gyrating component is absent.

On the other hand, a different form of accelerated ions is observed very close to the Martian quasi-perpendicular bow shock. Figures 6-8 show one such case in a traversal that is nearly normal to the average bow shock surface. Figure 6 shows the MEX orbit during 11:35 11:50 UT and the overview of ELS and IMA data during 11:30 12:06 UT on 12 July 2006 in the same format as Fig. 3 except that cylindrical coordinates with $R_{\mathrm{MSO}}\left(=\sqrt{Y_{\mathrm{MSO}}^{2}+Z_{\mathrm{MSO}}^{2}}\right)$ are used in the orbit plot, where the definition of the Mars-Sun Orbit (MSO) Cartesian coordinate system is the same as for VSO in Fig. 3 except that the reference planet is Mars instead of Venus. The ELS data show structured electrons in the same manner as in Fig. 3, indicating the connectivity to the bow shock during the entire period. The low-energy electron population observed between 11:52 11:57 UT is most likely caused by the spacecraft attitude system (attitude started to change at around 11:52 UT), adding a small amount of gas into the environment which is ionized.

Figure 7 shows the IMA proton data from azimuthal sectors $\phi=1 \sim 4$ for each elevation scan during 11:36 11:56 UT ( ix scans). The bottom panel is the summation of all azimuthal $(\phi)$ sectors and is essentially the same as the IMA panel of Fig. 6 except for the unit and the energy range. Each panel of Fig. 7 displays an elevation-energy spectrogram in 
$192 \mathrm{~s}$ using the same format as Fig. 4. The primary ring distribution (cycloid motion of newly ionized hydrogen with zero initial velocity) is marked by blue leftward pointing arrows and traced with blue dashed lines, by which the local $\boldsymbol{L}-\boldsymbol{M}-\boldsymbol{N}$ right-handed Cartesian coordinate is determined, where $N$ is the estimated IMF orientation. See Yamauchi et al. $(2006,2008)$ for definition and procedures for mapping IMA data into the $\boldsymbol{L}-\boldsymbol{M}-\boldsymbol{N}$ frame to determine the IMF orientation.

In addition, $L_{X}=0$ is imposed to fill the last degree of freedom in the minimum variance method (this method constrains five components out of the total six components of $\boldsymbol{L}$ and $\boldsymbol{M}$ ). Furthermore, the average $V_{N}$ is assumed to be zero for the primary ring ions (in practice, we manually tuned the $\boldsymbol{N}$ direction to satisfy this condition) because a newly ionized exospheric neutral has a zero initial velocity compared to the solar wind and a subsequent velocity at time $t$ :

$\left.\boldsymbol{V}=\left(\boldsymbol{V}_{\mathrm{SW}} \cdot \boldsymbol{M}\right)[(1-\cos (\Omega t)) \boldsymbol{M} \mp \sin (\Omega t) \boldsymbol{L})\right]$

without the $N$ component in the planetary rest frame, where $\Omega$ is the proton gyrofrequency. This additional condition reduces the error in calculating the $N$ direction, which is about $\pm 5^{\circ}$ during the first four scans and about $\pm 10^{\circ}$ afterward in this particular traversal.

The data in the instrument coordinates (Fig. 7) is converted to these $\boldsymbol{L}-\boldsymbol{M}-\boldsymbol{N}$ coordinates in Fig. 8. In both the $V_{L}-V_{N}$ projection (upper) and $V_{L}-V_{M}$ projection (lower), all velocity space points are marked when the registered counts are more than 5 counts in each measurement $(100 \mathrm{~ms})$ for a given elevation direction and energy (this threshold is increased to 10 counts per $100 \mathrm{~ms}$ for solar wind protons). The color of the marks shown in Fig. 8 corresponds to the color of arrows and traces shown in Fig. 7, so that one can relate the classification of ions shown in Fig. 8 to the raw data displayed in Fig. 7. Note that IMA does not cover all $4 \pi$ steradian, and that some of the viewing directions are blocked by the spacecraft. The invisible directions mostly lie at the lower part of the $V_{L}-V_{M}$ panels of Fig. 8 (the region of clustered points corresponds to $-45^{\circ}$ elevation or $\theta=0$ ). Due to this limitation, one must use information from both Figs. 7 and 8 when analysing these ions.

The IMF orientation is given as $\pm N$, and the $\boldsymbol{N}$ vector values in the MSO coordinate system are given in Fig. 8. The IMF orientation is stable during the first four scans (11:36 11:49 UT), and this stable orientation is drawn in Fig. 6 (upper panel). To analyze the ion motion near the bow shock, one may consider using the de Hoffman-Teller frame (the shock reference frame in which the injected plasma flows along the upstream magnetic field). However, the angle between $\pm N$ and the bow shock normal is very close to $90^{\circ}$, and the angle between $\pm N$ outward from the shock and the solar wind vector is much larger than $90^{\circ}$. These facts cause a large uncertainty in obtaining the velocity of de Hoffman-Teller frame (de Hoffman-Teller velocity). Furthermore, one may not use the de Hoffman-Teller frame when
MEX/ASPERA-3, 2005-7-12
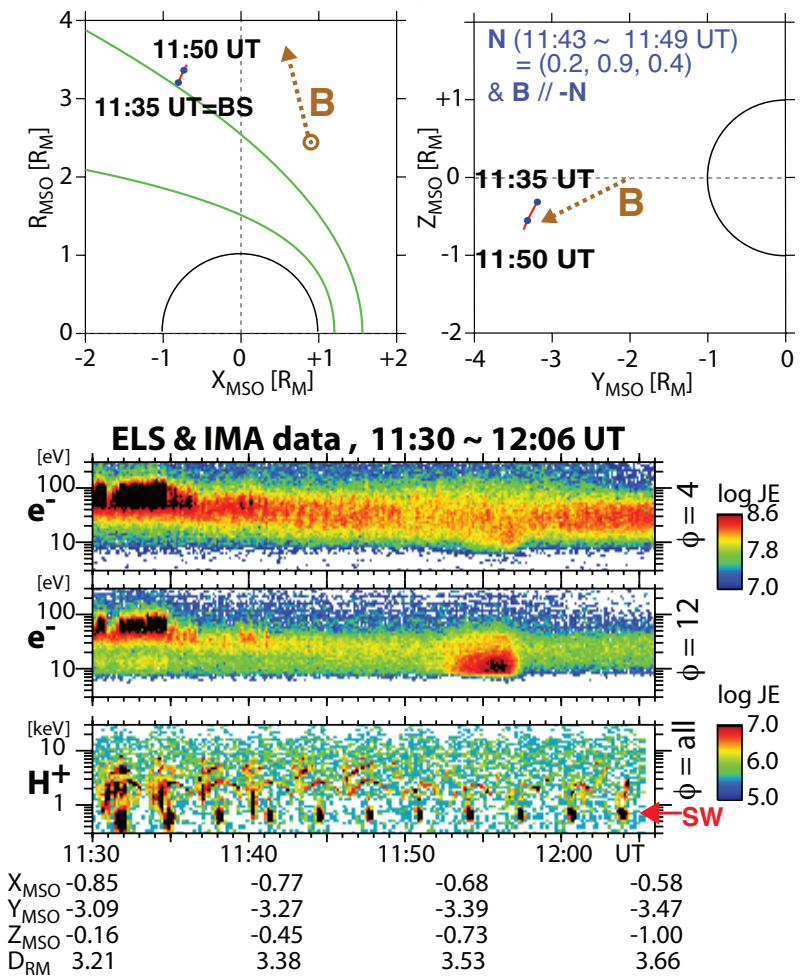

Fig. 6. Overview of the MEX orbit during 11:35 11:50 UT and hot plasma data during 11:30 12:06 UT on 12 July 2005. The format is the same as in Fig. 3 except that there is no magnetic field measurement and that cylindrical $R_{\mathrm{MSO}}=\sqrt{Y_{\mathrm{MSO}}^{2}+Z_{\mathrm{MSO}}^{2}}$ is used instead of $Y_{\mathrm{MSO}}$ in the top left panel. There, the average boundary positions (bow shock and induced magnetosphere boundary) are drawn with green lines. The unit $R_{\mathrm{M}}$ is the Mars radius $(3397 \mathrm{~km})$. The solar wind protons are seen at around $0.7 \mathrm{keV}$ in the proton panel (regularly repeating every $192 \mathrm{~s}$ ). The bow shock is recognized at around 11:35 UT, and multiple ring-like structures are recognized until around 11:50 UT. The projection of the magnetic field direction derived from the ring-like structure is drawn on the orbit plots in the top panels (see text).

the curvature of the shock cannot be ignored compared to the curvature of the ion motion (both gyromotion and motion along the magnetic field), as is the case for the subsolar bow shock region of Mars (cf. Table 1) or at cometary bow shocks. Nevertheless, we calculated the de Hoffman-Teller velocity $\boldsymbol{V}_{\mathrm{HT}}=\boldsymbol{n} \times\left(\boldsymbol{V}_{\mathrm{SW}} \times \boldsymbol{B}\right) /(\boldsymbol{B} \cdot \boldsymbol{n})$ to diagnose the ion motion with respect to the bow shock surface (see Table 2 for definition of vectors) because this particular MEX traversal is at the bow shock flank where the curvature of the shock is relatively small.

We assumed a simplified shock normal vector $\boldsymbol{n}=(+0.6$, $-0.8,0$ ) in the MSO coordinate system (cf. Fig. 6). Table 2 shows the calculated $\boldsymbol{V}_{\mathrm{HT}}$ as well as other key vector values during the second scan (11:39:36 11:42:48 UT) in both the MSO and the local $\boldsymbol{L}-\boldsymbol{M}-\boldsymbol{N}$ Cartesian coordinate 


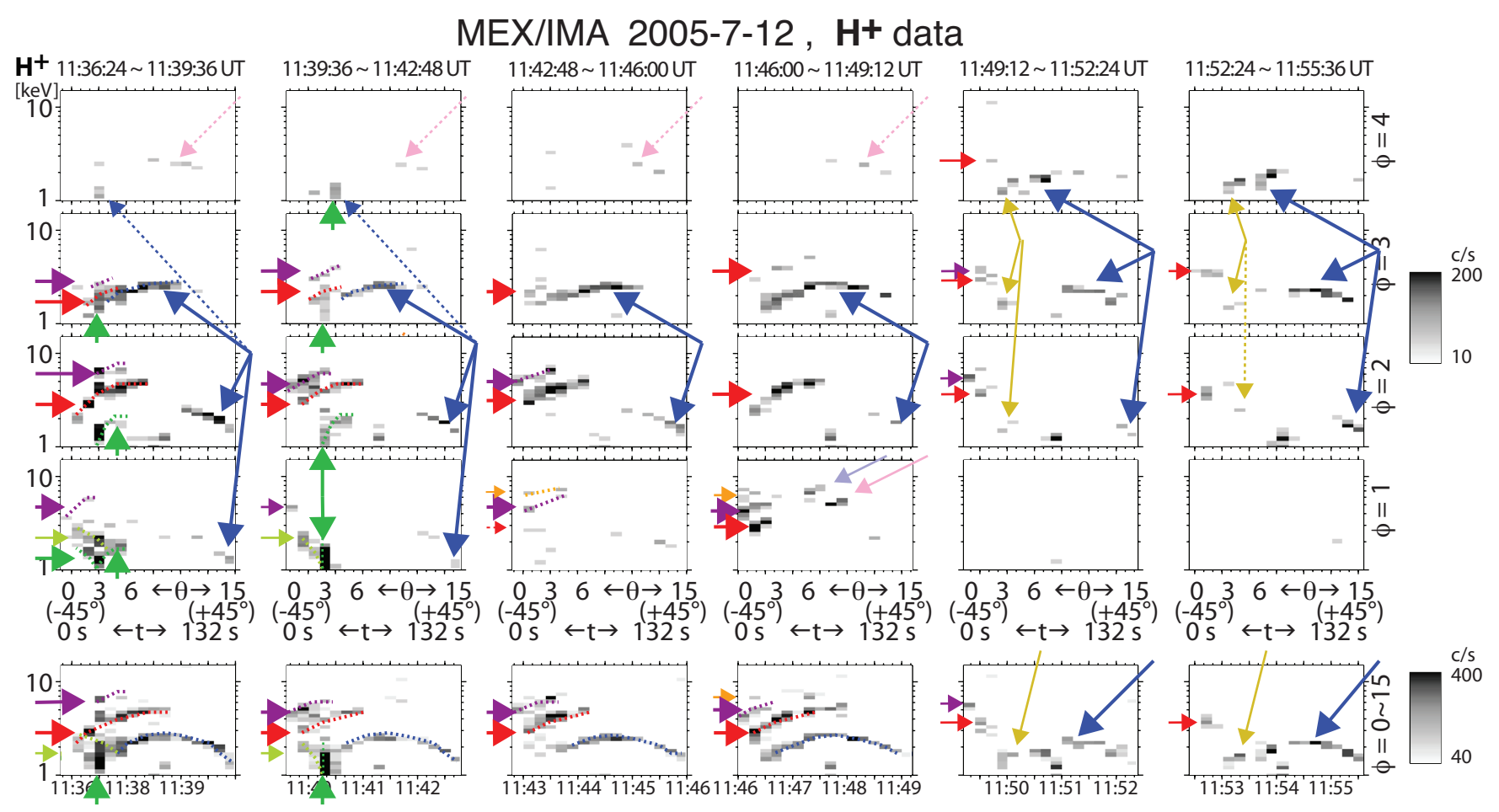

Fig. 7. Energy-time spectrograms from MEX/IMA proton channels ( $1 \sim 15 \mathrm{keV}$ per charge) on 12 July 2005 during 11:36:24 11:55:36 UT (part of Fig. 6) in a similar format as Fig. 4. Four different azimuthal sectors (from top, $\phi=4,3,2,1$ ) and integration over all $\phi$ are presented. sector 0 is not presented because it gathers contamination from all of the other sectors. The first pixels of each spectrogram correspond to $-45^{\circ}$ elevation $(\theta=0)$ and the last pixels to $+45^{\circ}$ elevation $(\theta=15)$. Contamination from solar wind alpha particles is seen at about $1.5 \mathrm{keV}$ per charge (i.e., $3 \mathrm{keV}$ with $q=2$ ) at $0^{\circ}$ elevation $(\theta=8)$. Otherwise, the ion species (proton) is confirmed by the energy-mass matrix (not shown here). Arrows with the same color indicate the same ion population and arrows with different colors indicate different ion populations. The same colors are used in Fig. 8, i.e., blue is the primary ring distribution, and red, purple, and orange are accelerated ions.

systems. The field-aligned velocity of the observed ions in the de Hoffman-Teller frame is obtained by simply subtracting the $N$ component of $\boldsymbol{V}_{\mathrm{HT}}$ from the $N$ component of the velocity values, and the sign of $n_{N}$ determines the direction of escape in the $\boldsymbol{L}-\boldsymbol{M}-\boldsymbol{N}$ coordinates. This subtraction is visualized in Fig. 8 by adding hatched areas: the velocity-space area that corresponds to nearly zero field-aligned velocity in the de Hoffman-Teller frame is hatched with light-grey colors, above and below which correspond to ions moving into the bow shock (grey hatch) and ions exiting from the bow shock (no hatch), respectively. The thickness of this lightgrey hatch area corresponds to $\pm 5^{\circ}$ errors that are assumed in the bow shock normal direction. The error associated with the uncertainty of the estimated magnetic field orientation lies within the light-grey hatch area. Note that the large $\left|V_{\mathrm{HT}}\right|$ value comes from the large angle between the $\pm \boldsymbol{N}$ outward from the shock normal and the solar wind vector (much larger than $90^{\circ}$ ), which is another large source of error.

\subsection{Two-layer structure}

Figures 7 and 8 show a smooth scan-to-scan transition of the location of the observed ions' velocity space. In these fig-
Table 2. Velocity components in different frames of reference.

\begin{tabular}{lcc}
\hline frame & $(X, Y, Z)$ & $(L, M, N)$ \\
\hline $\boldsymbol{L}$ & $\sim(0,+0.55,-0.8)$ & $(+1,0,0)$ \\
$\boldsymbol{M}$ & $\sim(-1.0,+0.15,+0.1)$ & $(0,+1,0)$ \\
$\boldsymbol{N}$ & $\sim(+0.2,+0.8,+0.55)$ & $(0,0,+1)$ \\
$\boldsymbol{B} / \boldsymbol{B}$ & $\sim(-0.2,-0.8,-0.55)$ & $(0,0,-1)$ \\
$\boldsymbol{n}$ & $\sim(+0.6,-0.8,0)$ & $\sim(-0.45,-0.7,-0.5)$ \\
$\boldsymbol{V}_{\mathrm{SW}} / V_{\mathrm{SW}}$ & $(-1,0,0)$ & $\sim(0,+1.0,-0.2)$ \\
$\boldsymbol{V}_{\mathrm{R}} / V_{\mathrm{SW}}$ & $\sim(-0.3,-0.95,0)$ & $\sim(-0.5,+0.2,-0.8)$ \\
$\boldsymbol{V}_{\mathrm{HT}} / V_{\mathrm{SW}}$ & $\sim(-1.2,-0.95,-0.6)$ & $\sim(0,+1.0,-1.3)$ \\
\hline $\boldsymbol{B}:$ interplanetary magnetic field & \\
$\boldsymbol{n}:$ bow shock normal & \\
$\boldsymbol{V}_{\mathrm{R}}:$ specular reflection of solar wind $\boldsymbol{V}_{\mathrm{SW}}$ \\
$\boldsymbol{V}_{\mathrm{HT}}:$ de Hoffman-Teller velocity \\
\end{tabular}

ures, one can recognize many ion counts that do not belong to the solar wind (light blue plus signs in Fig. 8) or the primary ring distribution (blue arrows in Fig. 7 and blue circles in Fig. 8). These extra counts are classified into two categories: energy-bunched multiple ring-like population at 


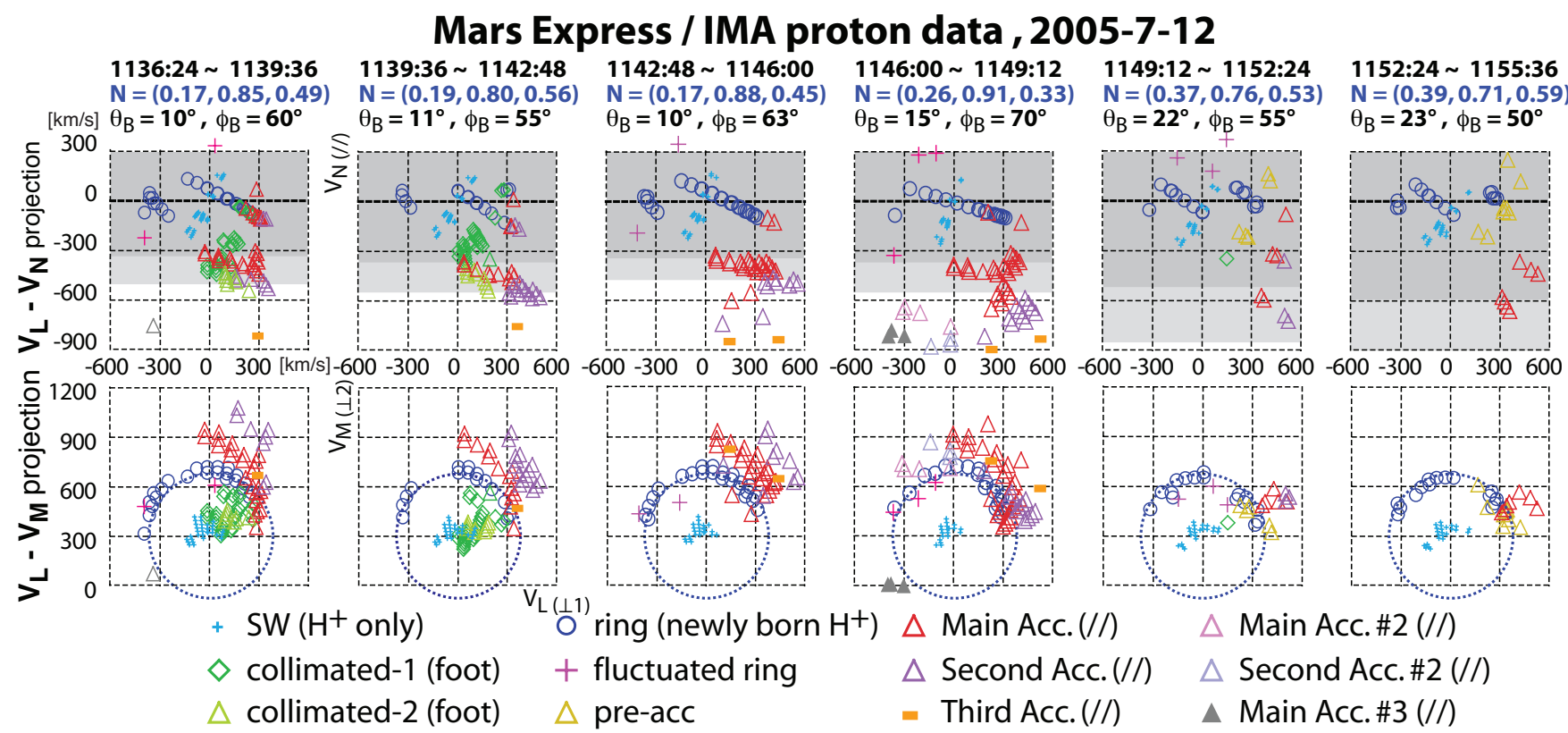

Fig. 8. Velocity scatter plots of the six consecutive scans shown in Fig. 7. The velocity space points with 5 counts or more during the $100 \mathrm{~ms}$ measurement period are plotted in the spacecraft reference frame (this threshold is increased to 10 counts per 100 ms measurement for the solar wind that is marked by light blue pluses). The local Cartesian coordinates $\boldsymbol{L}-\boldsymbol{M}-\boldsymbol{N}$ are derived such that $\boldsymbol{N}$ points along the magnetic field and $L_{X}=0$. The estimated $N$ vector values in the MSO coordinate are given at the top of each panel, where $\theta_{B}$ is the angle between the $\boldsymbol{N}$ vector and $\boldsymbol{Z}$, and $\phi_{B}$ is the rotation angle of the $\boldsymbol{N}$ vector in the $\boldsymbol{Y}-\boldsymbol{Z}$ plane stating from the $\boldsymbol{Z}$ axis (the same definition as Yamauchi et al., 2006, 2008). The grey-hatched area represents velocity space in which ions have positive $V_{N}$ in locally-defined de Hoffman-Teller frame, where the error bar (hatched by light grey) is defined by assuming the $\pm 5^{\circ}$ uncertainty in the bow shock normal direction. Identified ion populations use the legend at the bottom. The color-coded symbols corresponds to the color-coded arrows shown in Fig. 7.

higher energy than the primary ring distribution (marked red, purple, or orange in Figs. 7 and 8) and a collimated population in a narrow elevation angle but broad energy range (marked green in Figs. 7 and 8). The former is continuously found during the first four scans (five scans from the bow shock crossing according to Fig. 6) beyond which the intensity suddenly dropped, whereas the latter is found only during the first two scans (three scans from the bow shock crossing according to Fig. 6). Considering the spacecraft velocity with respect to the bow shock, five scans $(16 \mathrm{~min})$ correspond to a distance of about $700 \mathrm{~km}$ from the bow shock, whereas three scans $(9 \mathrm{~min})$ correspond to a distance of about $400 \mathrm{~km}$ from the bow shock. Such a double transition is commonly found in the other MEX bow shock traversals when multiple ring-like distributions are observed. Therefore, the observation indicates a spatial structure, i.e., a two-layer structure in the bow shock foot region. Since the gyroradius of $2 \mathrm{keV}$ protons in a $6 \mathrm{nT}$ magnetic field is about $1000 \mathrm{~km}$ and the proton inertia length for a $5 \mathrm{~cm}^{-3}$ proton plasma is about $100 \mathrm{~km}$, the distance of $400 \mathrm{~km}$ and the distance of $700 \mathrm{~km}$ are both within the range of possible thickness of a foot region of the bow shock.

According to Fig. 8, the collimated population that is specific to the first layer (closer layer to the bow shock) extends from $\boldsymbol{V}_{\mathrm{HT}}$ in velocity space and has a large field-aligned velocity $\left(V_{N} \sim-350 \mathrm{~km} \mathrm{~s}^{-1}\right)$. The energy-bunched multiple ring-like population that is found both in the first and second layers has $V_{\perp}$ in the solar wind frame (radial separation from the solar wind in the $V_{L}-V_{M}$ projection) slightly larger than the primary ring distribution, and has a large field-aligned velocity $\left(V_{N}=-350 \sim-700 \mathrm{~km} \mathrm{~s}^{-1}\right)$. The flow directions of both populations point away from the subsolar region. The field-aligned tailward flow with substantial gyromotion of both populations is similar to the reflected-gyrating component in the terrestrial bow shock foot region (Paschmann et al., 1981; Sckopke et al., 1990; Meziane, 2004a). It is worth reminding ourselves that the elevation-azimuth pattern of the gyrating ions as shown in Fig. 4 and that in Fig. 7 are quite different, echoing the conclusion for the Earth where the gyrating ions with large field-aligned velocity are of different origin in the distant foreshock than in bow shock foot region.

\subsection{Collimated tailward ion flow}

Let us examine the collimated population that is specific to the first layer. In the first two scans in Fig. 7, this population (marked by green vertical arrows) shows a high count rate in a narrow elevation angle range $\left(\theta=3\right.$, less than $15^{\circ}$ wide) and at energies $<2 \mathrm{keV}$. A similar ion population has been reported in Yamauchi et al. (2006) where no solid explanation was given. Found at exactly the same elevation angle for two consecutive scans, these ions do not likely show a temporal 
variation and more likely show spatial layer concept as mentioned in the previous subsection. In Fig. 8, this population (green diamonds) occupies the same $V_{L}-V_{M}$ velocity space as another population that is marked by light green triangles (they correspond to light green right arrows in Fig. 7).

Since Fig. 8 is very busy with many different populations at similar locations in velocity space, all ion populations during the second scan (11:39:36 11:42:48 UT) are schematically summarized by solid lines in Fig. 9a and $b$ in the same format, colors, and coordinate system as used in Fig. 8. Velocity values in the MSO coordinate are given for each branch in this figure. In Fig. 9a, one can see a systematic difference between the green- and light green-marked branches. The green branch extends from $\boldsymbol{V}_{\mathrm{HT}}$ (point $\mathrm{S}$ : corresponding to $\phi=1$ in Fig. 7) to nearly perpendicular flow with respect to the IMF, reaching to the same location in velocity space as the primary ring ions (point $\mathrm{E}$ : corresponding to $\phi=4$ in Fig. 7). The light green branch also extends from $V_{\text {HT }}$ but to higher $\left|V_{\|}\right|$. Note that the lower cutoff of the green branch in the $\left|V_{\|}\right|$direction of Fig. 9 (corresponding to the lower energy cutoff in Figs. 6 and 7) is not due to any instrumental limit because Fig. 6 shows that this signature is absent below $1 \mathrm{keV}$ at around 11:37 UT. Having the similar velocities as $\boldsymbol{V}_{\mathrm{HT}}$ for both branches means that ions of these branches stay in the vicinity of the bow shock while they flow tailward at speeds comparable to or faster than the solar wind velocity.

\subsection{Energy-bunched gyrating ions}

We next examine the energy-bunched multiple ring-like population that is found both in the first and second layers, i.e., up to about $700 \mathrm{~km}$ from the bow shock. MEX has often observed such accelerated ions at higher energy than the primary ring distribution (Dubinin et al, 2006; Yamauchi et al., 2008). In Fig. 7, this population is seen as several different stripes above the primary ring distribution in the energy domain for a given direction (azimuth $\phi$ and elevation $\theta$ ), as marked by right arrows in orange, purple, and red colors from the highest to the lowest energies. Let us take a scan during 11:46:00 11:49:12 UT for further examination.

The primary ring distribution is observed mainly in azimuthal sector $\phi=3$ at energies $<3 \mathrm{keV}$. In the same sector $(\phi=3)$, another distribution is seen with an energy $>3 \mathrm{keV}$ as marked by a red right arrow at $\theta=1,3,4$, and 6 . This population is clearer in sectors $\phi=2$ and $\phi=1$ at the same energy ( $2.5 \sim 5 \mathrm{keV})$, and is also marked by red right arrows. In the first four elevations $(\theta=0,1,2,3)$ in sector $\phi=1$, two more independent branches are observed at higher energies (marked by purple right arrows at around $4 \sim 5 \mathrm{keV}$, and by orange right arrows at around $7 \mathrm{keV}$ ) than the red-marked branch. These three different branches of accelerated ions are all protons according to the energy-mass matrix (not shown here), and are plotted using red empty triangles, purple empty triangles, and orange bars, respectively, in Fig. 8 where the primary ring distribution is plotted using blue circles. The
2005-7-12 example (11:40 UT)

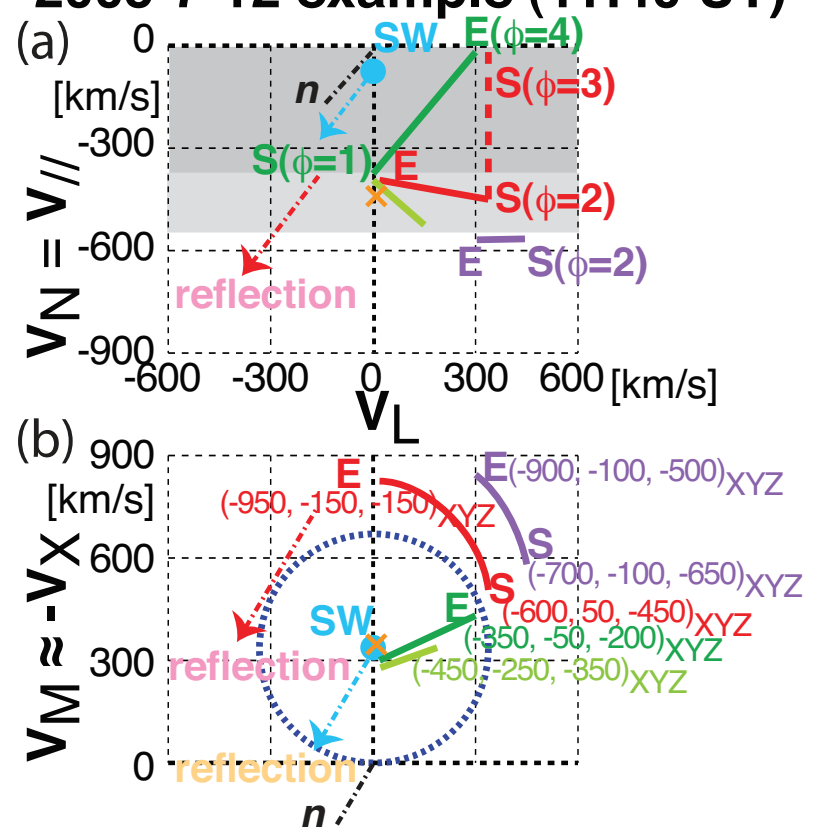

(c)

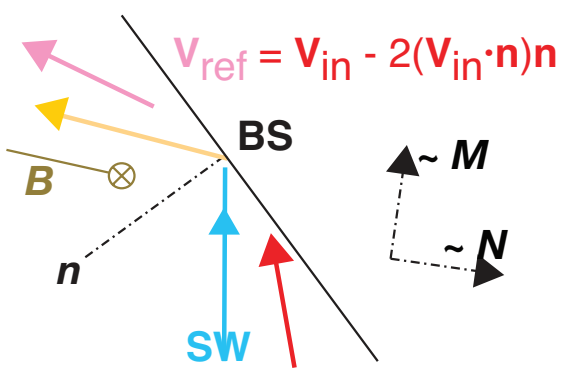

Fig. 9. Illustration of velocity space trajectory in the $\boldsymbol{L}-\boldsymbol{M}-\boldsymbol{N}$ coordinate depicted from the 11:39:36 11:42:48 UT data (second scan) of Fig. 8, where $N=(+0.2,+0.8,+0.55)$ is the magnetic field orientation that is estimated from the ring distribution (light-blue dashed circle. The local bow shock (BS) normal direction $(\boldsymbol{n})$ is assumed to be $(+0.6,-0.8,0)$ in MSO coordinates, and is drawn with dash-dotted lines. (a) $L-N$ projection of velocity space ion trajectories at around 11:40 11:41 UT. (b) $L-M$ projection of velocity space ion trajectories at around 11:40 11:41 UT. (c) The local BS configuration in MSO coordinates $\left(+\boldsymbol{X}_{\text {MSO }}\right.$ points downward and

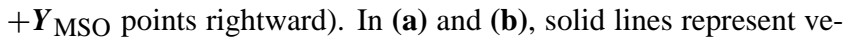
locity space trajectory during the gyration (start and end point in the observed phase-space are marked as $\mathrm{S}$ and $\mathrm{E}$, respectively under assumption of $\boldsymbol{B} / /-\boldsymbol{N}$ ), whereas dash-dotted arrows represent jumps in velocity due to specular reflection at the bow shock. Point $S$ of the red and purple lines correspond to the starting elevation angle $(\theta=0$ in Fig. 2), and the quadrant below $S$ in (b) is outside the field-of-view of IMA. The orange cross indicates the de HoffmanTeller velocity $\boldsymbol{V}_{\mathrm{HT}}=\boldsymbol{n} \times\left(\boldsymbol{V}_{\mathrm{SW}} \times \boldsymbol{B}\right) /(\boldsymbol{B} \cdot \boldsymbol{n})$. The value of $\phi$ in the $L-N$ projection is the azimuthal sector number that recorded the ions in Fig. 7. The ion trajectories marked by the green and light green lines in (a) and (b) corresponds flows nearly along the BS, and the start point of the green line overlaps with $\boldsymbol{V}_{\mathrm{HT}}$. 
energy ratio between the red-marked branch and the purplemarked branch is about 3:5. This suggests that the multipleenergy structure of ions upstream of the Earth reported by Lutsenko and Kudela (1999) and Klassen et al. (2009) might not be due to different species but related to multiple acceleration.

The corresponding ion population in the other scans is also subdivided into red-, purple-, and orang-marked branches such that their velocity distribution is consistent between neighboring scans. The bottom panels in Fig. 7 support the classification; e.g., the primary acceleration (red-mark) is seen throughout all 6 scans at the same energy. In this way, the variation of the accelerated ions every 192-s scan is also obtained. For example, clustering patterns (in direction and energy) are smoothly changing between scans in Fig. 7 except for a sudden decrease in the count rate of the accelerated ions at around 11:49 UT (between the fourth and fifth scans) when the estimated IMF orientation changed about $20^{\circ}\left( \pm 10^{\circ}\right)$. After this time, the count rate of the accelerated ions is much lower than that of the foreshock ions observed by VEX. The drop of the accelerated ions within a short distance from the bow shock is a common feature as mentioned in Sect. 4.1, while the change of IMF orientation can be another factor that contributed to the sudden drop for this particular traversal.

Back to the fourth scan (11:46:00 11:49:12 UT) in Fig. 8, all three branches of accelerated ions have similar finite $V_{\perp}$ in the solar wind frame, whereas they have quite different $V_{\|}\left(\right.$or $V_{N}$ ). The red empty triangles in Fig. 8 (most intense branch in Fig. 7 marked by red right arrows) have fieldaligned speed of about $400 \mathrm{~km} \mathrm{~s}^{-1}$ which is comparable to $\left|V_{\mathrm{HT} \|}\right|$, the purple empty triangles (purple right arrows in Fig. 7) about $700 \mathrm{~km} \mathrm{~s}^{-1}$, and the orange bars (orange right arrows in Fig. 7) about $900 \mathrm{~km} \mathrm{~s}^{-1}$. The first three scans in Fig. 8 show a larger separation in $V_{\|}$than in $V_{\perp}$ between different branches and the near-zero field-aligned speed of the red branch in the de Hoffman-Teller frame. Thus, the bunching in the energy domain (Fig. 7) is attributed to a bunching in the field-aligned velocity (Fig. 8).

In the $V_{L}-V_{M}$ plot of Fig. 8, all these accelerated ions are found outside of the dashed light-blue circle which represents the trajectory of pick-up ions. This means that these accelerated ions are actually gyrating with slightly larger $V_{\perp}$ than $V_{\mathrm{SW} \perp}$ in the solar wind frame, and do not represent a field-aligned beam. In the planetary frame, these ions flow mainly in the $-X_{\mathrm{MSO}}$ and $-Z_{\mathrm{MSO}}$ direction. The first branch (red arrow in Fig. 7 and red empty triangle in Fig. 8) has $V_{X}=-600 \sim-900 \mathrm{~km} \mathrm{~s}^{-1}$ and $V_{Z}=-400 \sim-200 \mathrm{~km} \mathrm{~s}^{-1}$. The second branch (purple arrow in Fig. 7 and purple empty triangle in Fig. 8) has $V_{X}=-700 \sim-1000 \mathrm{~km} \mathrm{~s}^{-1}$ and $V_{Z}=-600 \sim-500 \mathrm{~km} \mathrm{~s}^{-1}$ until 11:49 UT. Their tailward velocity is much larger than the solar wind velocity $\left(V_{\mathrm{SW}} \sim\right.$ $350 \mathrm{~km} \mathrm{~s}^{-1}$ ).
In addition to the three branches of accelerated ions, the fourth scan (11:46:00 11:49:12 UT) in Fig. 7 shows two other populations in the center elevation $(\theta=6,7,8,9)$ of the first azimuth sector $(\phi=1)$. They are plotted using light red and light purple triangles in Fig. 8. They have the same characteristics as the above-mentioned accelerated ions (red and purple triangles), but they appear disconnected from the main accelerated population in velocity space.

\subsection{Gyrophase bunching}

Unlike the primary ring distribution, the energy-bunched accelerated ions are found only within a limited phase angle range of gyration in the solar wind frame in Figs. 8 and $9 b$ for all three branches. The limitation in the extent of gyrophase is more prominent for the higher energy population. Although the limited field-of-view of IMA makes it difficult to identify the starting points of these populations in velocity space, the end points are within the IMA field-of-view (cf. Fig. 7). The branches of energy-bunched accelerated ions do not extend to higher elevation $\theta$ (elevations roughly correspond to gyrophase angles).

The count rate (which is nearly proportional to energy flux) of these branches gradually decreases from $\theta=0$ to higher $\theta$, although the flux is expected to be constant over gyrophase. The extension in phase in Figs. 7 and 8 is rather constant during the first four scans at different distances from the bow shock, indicating that gyrophase bunching might be related to the bow shock structure or reflection condition at the bow shock. The decrease in the count rate from $\theta=0$ to higher $\theta$ in Fig. 7 suggests that the direction of gyration is also from $\theta=0$ to higher $\theta$ (Yamauchi et al., 2008). This direction corresponds to a counter clockwise gyration in the $V_{L^{-}} V_{M}$ plane of Fig. 8, i.e., to IMF polarity in the $-\boldsymbol{N}$ direction.

\section{Discussion}

\subsection{Venus foreshock}

When the VEX position is magnetically connected to the Venus bow shock, VEX observes ion populations similar to those observed in the terrestrial foreshock under similar IMF conditions. The observed ion populations show large $V_{\|}$and limited $\delta V_{\|}$, which cannot be explained by a velocity filter effect. It rather indicates that the population experienced either a field-aligned potential acceleration or specular reflection. To show the energization and motion of specularly reflected ions and the other ions in the planetary frame, Fig. 10 schematically illustrates the trajectories of specularly reflected ions immediately upstream of the quasi-perpendicular bow shock in the planetary frame. Figure 10a corresponds to the subsolar region where the curvature is largest and Fig. 10b corresponds to the bow shock 
(a) Subsolar Bow Shock

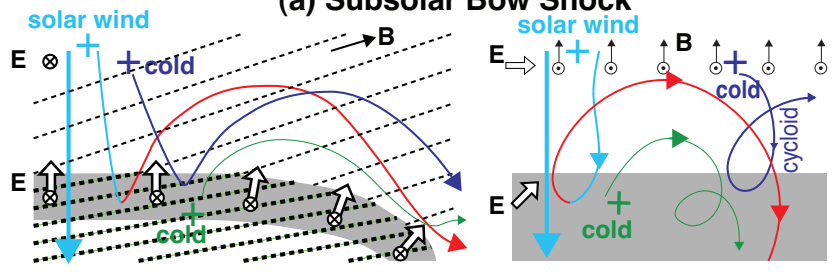

(b) Flank Bow Shock

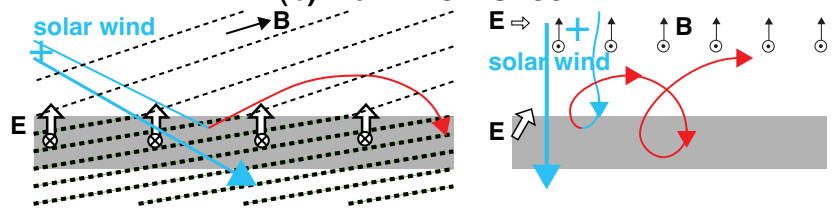

Fig. 10. Schematic illustration of ion motions just outside a quasiperpendicular bow shock (grey area) with a finite magnetic field component normal to the shock surface. (a) Subsolar bow shock where the curvature of the bow shock cannot be ignored. (b) Flank bow shock where the reflection direction can easily be field-aligned. Left panels show the projection of ion trajectories onto a plane which contains the IMF $(\boldsymbol{B})$ and solar wind, and right panels show the projection of the same ions onto a plane that is perpendicular to the left panel's plane (depicting gyro-cycloid motions). Reflection of ions at the bow shock, which is due to the combination of outward electric field and change of the magnetic field, is assumed to be nearly specular. Note that a specular reflection is impossible in simple 1-D shock models with DC electric and magnetic fields observed at the Earth, and hence, the ion motion inside the bow shock is an open question. With this limitation, three types of source ions are illustrated. If an incoming ion is specularly reflected, the ion energy is conserved in the bow shock reference frame, but it increases in the solar wind reference frame, resulting in a shift of the guiding center in the interplanetary electric field direction. The particle's energy gain is proportional to the gyroradius, which is proportional to the guiding center velocity. A local ion from inside the shock ramp (green line) gains $V_{\|}$by electrostatic acceleration across the shock. A newly ionized exospheric neutral (blue lines) has zero field-aligned velocity in the planetary frame. Since the incoming solar wind (light blue lines) has a large angle with respect to the IMF for a quasi-perpendicular shock, conversion of the incident flow to the field-aligned direction by specular reflection is more prevalent along the frank (b) than near the subsolar region (a). The flank is also favorable for multiple bouncing (i.e., multiple accelerations to higher energy) because of less curvature.

flank where the curvature is much smaller than in the subsolar region. In Fig. 10, ion motion in the shock ramp is not predictable because of the turbulent field configuration, and we have simply connected the ion trajectories of the incoming and reflected ions by assuming specular reflection. Note that a specular reflection in the rest frame of the shock does not look like a specular reflection in the solar wind frame or de Hoffman-Teller frame. Therefore, the shock rest frame is employed in Fig. 10.
As illustrated in the right panels of Fig. 10, specular reflection means a shift of guiding center in the direction of the $-\boldsymbol{V} \times \boldsymbol{B}$ electric field (Alfvén and Fälthammar, 1963), and also means that a portion of the ion energy is converted from gyrating motion into field-aligned motion as illustrated in left panels of Fig. 10 (Gosling et al., 1982; Meziane et al., 2004a). However, the latter type of the energy conversion is facilitated only at the bow shock flank where solar wind ions enter the shock obliquely to its surface (Fig. 10b), but not at the subsolar bow shock where solar wind ions enter nearly normal to its surface (Fig. 10a). To overcome this difficulty at the subsolar bow shock, a ripple structure of the shock (Meziane et al., 2004a) due to, e.g., ion cyclotron wave excitation (Winningham et al., 2006), has been proposed, although such a structure has only been examined remotely (Miao et al., 2009) but not by in-situ observations.

\subsection{Absence of foreshock ions at Mars}

Contrary to Venus, similar ion distributions are not observed upstream of the Martian bow shock by MEX for distances beyond one ion gyroradius from the bow shock. This difference most likely comes from the absence of the gyrating component of the foreshock ions. On the other hand, using single traversal data from the Phobos-2 spacecraft, Barabash and Lundin (1993) found ion signatures which are consistent with field-aligned beams while they did not find any signature of the gyrating component (the field-of-view of the instrument does not cover all phase angles, though). Dubinin et al. (1995, 2000), also using the Phobos-2 data, reported foreshock signatures in density and wave activity in the upstream region that is magnetically connected to the bow shock. These observations indicate two possibilities: without the magnetic field data, MEX simply cannot identify the field-aligned beams that occupy only one sector and elevation angle range; or the field-aligned beams in foreshock region of Mars are much less intense than those of Venus or the Earth.

Let us examine the first case. Although a large part of the gyrating component is considered to be generated through coherent wave disruption of the field-aligned beams, i.e., at some distance from the bow shock (Fuselier et al., 1986), this does not explain the MEX observation because the foreshock signature that is similar to Figs. 3-5 is observed already at several gyroradii $(<500 \mathrm{~km})$ distance from the Venus bow shock (not shown here). Barabash and Lundin (1993) proposed that the small bow shock size of Mars normalized by the ion gyroradius does not allow enough space for the necessary instabilities to develop, which convert the field-aligned beams into gyrating components. Examination of this scenario requires a wave experiment, which is not present on MEX.

The second case means that ion acceleration, including reflection, at the bow shock is different between Mars on one hand and Venus or the Earth on the other hand. In fact, the 
bow shock foot region of Mars is different from that of the Earth; e.g., Mars generates an unexpected two-layer structure that the Earth does not have. This difference most likely comes from the difference in the plasma parameters as mentioned in the introduction: the bow shock size (radius of curvature) compared to the proton gyroradius, and the cold ion abundance in the bow shock region. These effects are discussed in the following Sects. 5.3, 5.5, and 5.7.

\subsection{Finite curvature effect}

In the beginning of Sect. 4, we mentioned a few conditions that could partly invalidate a de Hoffman-Teller analysis. The validity of a de Hoffman-Teller frame is directly related to the chance of the second reflection. Let us take a proton from the red-marked branch in Figs. 7-9. It has a gyroradius $\left(r_{\mathrm{g}}\right) \sim 1000 \mathrm{~km}$ in a $5 \mathrm{nT}$ IMF whereas the bow shock size is about $\sim 5000 \mathrm{~km}$ for Mars. The trajectory of such an ion in the planetary frame is drawn by red lines in Fig. 10. Note that one may not use de Hoffman-Teller frame in examining any effects that may invalidate the assumptions made.

The right panels of Fig. 10 show the gyromotion of reflected ions: their return positions at the bow shock are roughly $2 r_{\mathrm{g}}$ away from the original reflection points. The distances to the return positions of the reflected ions along the bow shock in the projection perpendicular to the interplanetary electric field (left panels) can be larger than $2 r_{\mathrm{g}}$ if the reflected ions have substantial field-aligned velocities after the reflection. Therefore, the curvature radius of a reflected ion trajectory is more than $2 r_{\mathrm{g}}$ (more than $2000 \mathrm{~km}$ in the present example). If this scale length is about the same size as that of the bow shock curvature radius as illustrated in Fig. 10a (e.g., at the subsolar bow shock of Mars), this ion may not hit the bow shock after completing half a gyration when the curvature is positive. In this way, the assumption of a planar shock (mandatory for de Hoffman-Teller frame) becomes questionable. We call this the "shock-disappearance effect" within this paper.

The "shock-disappearance effect" generally makes it easier for ions to escape and prevents multiple reflections at the bow shock. Therefore, the ions that have near zero fieldaligned velocity in the de Hoffman-Teller frame (e.g., red triangles in Fig. 8) may escape from the subsolar bow shock. Since the "shock-disappearance effect" is directly related to the scale length of the ion motion, the effect is more prominent for ions with high energies. For specularly reflected solar wind ions, this effect might be substantial along the entire dayside bow shock rather than only at the subsolar region.

\subsection{Multistep parallel acceleration}

The accelerated ions very close to the Martian bow shock have an unexpected feature, i.e., multiple-step acceleration in the magnetic field direction. This is seen as several discrete branches (marked red, purple, and orange) in both the energy domain shown in Fig. 7 and velocity space shown in Fig. 8 (see also Fig. 9). Such a bunching in the energy domain suggests, contrary to what the "shock-disappearance effect" suggests for the Mars, multiple reflections of the same ions at the bow shock because each reflection means a steplike gain of energy as mentioned in the introduction and in Sect. 5.1.

Dubinin et al. (2006) suggested that such multiple bouncing at the bow shock might explain their MEX observation, although no solid calculation was made. Note that Dubinin et al. called this the surfing model (Sagdeev, 1966; Lee et al., 1996; Zank et al., 1996) because the principle is the same. The simplest multiple-bouncing model is two-dimensional in the plane perpendicular to the magnetic field, and such a model explains the energy bunching in the $V_{\perp}$ domain but not in the $V_{\|}$domain. Considering the fact that specular reflection can convert energy from the perpendicular direction to the parallel direction with respect to the IMF in the shock rest frame (cf. Sect. 5.1), the multiple-bouncing model with specular reflection may explain the bunching in the $V_{\|}$domain.

Let us examine this scenario in the present case with the IMF direction and the average bow shock shape as illustrated in Fig. 6. Here, we use data corresponding to Fig. 9 (11:39:36 11:42:48 UT) and assume a specular reflection with respect to an assumed bow shock normal direction $\boldsymbol{n}$ $=(+0.6,-0.8,0)$ in the MSO coordinate system. For a surface with a normal vector $\boldsymbol{n}$, the velocity of specular reflection $\boldsymbol{V}_{\text {ref }}$ is calculated as $\boldsymbol{V}_{\text {ref }}=\boldsymbol{V}_{\text {in }}-2\left(\boldsymbol{V}_{\text {in }} \cdot \boldsymbol{n}\right) \boldsymbol{n}$, where $\boldsymbol{V}_{\text {in }}$ is the injection velocity. In the present case, the $N$ component of the solar wind increased from $-0.2 V_{\mathrm{SW}}$ (injection) to $-0.8 V_{\mathrm{SW}}$ (reflection); i.e., the flow is converted from nearly perpendicular flow to nearly parallel flow with respect to the estimated IMF in the shock rest frame by specular reflection as illustrated by the yellow arrow in Fig. 9c. In the solar wind frame, the perpendicular energy increases by this reflection as mentioned in the introduction.

In velocity space (Fig. 9a and b), this reflection means a jump in the trajectory, as drawn using the light blue dashdotted arrows. Once the reflected ions enter the solar wind electric field, these ions follow simple gyromotion in the solar wind frame, tracing a circular trajectory that is centered about the solar wind in Fig. 9b with constant $V_{N}$. In the shock rest frame, this means gain of kinetic energy in the perpendicular direction with respect to the magnetic field together with change in direction from dawnward to antisunward. Unfortunately, a large part of this partial circular trajectory in velocity space is outside of IMA's field-of-view from its beginning (end points of the light blue dash-dotted arrows) until near point $\mathrm{S}$ of the red line.

Point $\mathrm{E}$ of the red solid line in Fig. 9b has velocity of $\left(-950 \mathrm{~km} \mathrm{~s}^{-1},-150 \mathrm{~km} \mathrm{~s}^{-1},-150 \mathrm{~km} \mathrm{~s}^{-1}\right)$ in the MSO coordinate system in the planetary frame, and is approaching the bow shock as illustrated by the red arrow in Fig. 9c. Since the field-aligned velocity of the red branch in the de 
Hoffman-Teller frame is nearly zero, guiding centers of the relevant ions move nearly along the bow shock, which means that ions exiting from the shock return to the shock after one gyration. In fact, the red branch with flow direction approaching the bow shock is observed from immediately outside the bow shock, indicating that the ions of this branch will hit the bow shock.

If a substantial portion of the incoming ions that belong to the red branch is specularly reflected again at the bow shock as indicated by the red (injection) and pink (reflection) arrows in Fig. 9c, this reflection again converts the perpendicular energy to parallel energy with respect to the magnetic field in the shock rest frame. In velocity space (Fig. 9a and b), this reflection again means a jump in the trajectory, as drawn by the red dash-dotted arrows. The rest of the velocity space trajectory is similar to the one after the first reflection, except for a different $V_{\|}$that corresponds to the purple lines in Fig. 9a. Ions of this branch approach the bow shock at point $\mathrm{E}$, with the possibility of yet another reflection.

Here, we did not take into account the difference in the electric and magnetic fields between the foot region and the solar wind. Therefore, the observation is expected to slightly differ from the simplified trajectories. Yet, both the red and purple lines in Fig. 9b lie at close to the expected locations in velocity space, i.e., only tens of $\mathrm{km} \mathrm{s}^{-1}$ outside the blue dashed circle for both the first reflection and the red lines, and about a hundred $\mathrm{km} \mathrm{s}^{-1}$ outside the blue dashed circle for both the second reflection and the purple lines.

\subsection{Escape condition}

Unlike the red branch, the purple branch has a larger fieldaligned speed than $V_{\mathrm{HT} \|}$, indicating that the guiding center escapes from the bow shock into interplanetary space. The finite curvature of the bow shock makes this escape easier than what a de Hoffman-Teller analysis would indicate. However, the gyroradius of the purple-marked ions is as large as $1000 \mathrm{~km}$ in a $5 \mathrm{nT}$ magnetic field (recall that the field strength is not measured by MEX), whereas half the gyroperiod is only $6 \mathrm{~s}$ for the same condition. Therefore, the escape is not fast enough, so that a substantial fraction of these ions reach the bow shock again during their gyromotion. This explains why we observe the purple branch immediately outside the bow shock at the first scan in Figs. 7 and 8.

No organized ion branch is recognized at higher energies except the weak signature of the orange-marked branch. IMA's field-of-view allows detection of ions at a gyrophase that corresponds to approach toward the bow shock but not leaving from the bow shock. Thus, the absence of highly accelerated ions close to the bow shock agrees with the escape of the ions although it may also be partly due to the limited IMA sensitivity beyond $15 \mathrm{keV}$. Summarizing these results, the solar wind ions in the present case need to be reflected at least twice and often three times before they completely escape into interplanetary space without further reflections.
We do not know quantitatively whether such multiple reflections are generally required for ions to escape from the Martian bow shock and the other bow shocks such as Venus and the Earth. Using a particle simulation, Oka et al. (2005) suggested that multiple reflections at the shock are required to form the foreshock ions in the terrestrial case. If this is true, the Martian foreshock is formed mainly tailward of the subsolar point and is constrained very close to the shock because the finite curvature of the Martian bow shock decreases the chance of re-entry of reflected ions to the bow shock (cf. Sect. 5.3) and shifts the secondary reflection tailward rather than sunward (cf. Fig. 10a). This agrees with the lack of an ordinary foreshock in the MEX observation. On the other hand, the elevation-azimuth pattern of the observed ion branches is quite different from that of ordinary foreshock ions at Venus (Fig. 4). This issue needs future investigations.

\subsection{Foot region}

Close to the Martian quasi-perpendicular bow shock, two types of ion populations are observed in two layers: the green-marked branch within the first layer (nearest layer from the bow shock) and the red- and purple-marked branches in both the first and the second layers in Figs. 7-9. Both populations are gyrophase bunched and similar to those observed in the shock foot region (Meziane et al., 2004a). Paschmann et al. (1981) and Sckopke et al. (1983) showed that the ion distribution in the upstream foot region of a quasi-perpendicular bow shock is consistent with a partial ring distribution that originates from the reflected solar wind at the bow shock. Later, AMPTE and Cluster observations demonstrated that the ring distribution exists only within a few hundred km upstream of the bow shock (Sckopke et al., 1990; Möbius et al., 2001).

These characteristics agree well with the green-marked branch and its location, whereas the energy-bunched structure of the red- and purple-marked populations is specific to Mars. We are not aware of a similar energy-bunched structure at Venus. We are not aware of any report describing multistep acceleration at the terrestrial bow shock with the same intensity as in the Martian case. Therefore, the greenmarked population most likely corresponds to the ordinary foot ions rather than the red- and purple-marked population.

\subsection{Role of cold ions}

Why is multistep acceleration observed only at Mars? Why is a foreshock absent in the Martian upstream region? As mentioned in the introduction, the main differences between Mars and the Earth are the size of the gyroradius compared to the bow shock (cf. Table 1) and the cold ion abundance near the bow shock. The effect of finite curvature of the bow shock during ion bouncing may account for the second question as mentioned Sect. 5.5, but this effect unlikely answers 
the first question because the finite curvature reduces rather than increases the chance of re-entry of reflected ions, as illustrated in Fig. 10 (left panels).

However, the abundant cold ions at the location of the Martian bow shock may explain the Martian peculiarity because cold ions may influence the electric potential drop across the shock. Due to unsteady bow shock motion relative to the spacecraft, electric field measurement is very difficult at the terrestrial bow shock. Therefore, the observed potential drop across the terrestrial bow shock of only a few hundred volt (e.g., Eastwood et al., 2007, and references therein) does not exclude the possibility of localized and intermittent potential drops as large as one to a few $\mathrm{kV}$. In such a case, the bow shock electric field may cause specular reflection. Let us examine the consequence of such a scenario.

First, this scenario would allow single reflections of solar wind protons to form the foreshock upstream the subsolar bow shock only for the Earth and Venus but not for Mars because of the smaller electric potential drop across the Martian bow shock than those for the Earth or Venus due to the neutralizing cold ions. Second, the kinetic energy of the solar wind ions normal to the bow shock becomes an important factor that determines whether the incoming ions will be reflected or not. In this case, the solar wind is easier to be reflected when it enters obliquely to the shock surface (bow shock flank) as shown in Fig. 10b. At the same time, obliquely reflected ions do not easily escape with a single reflection, as is discussed in Sect. 5.5. Third, gyroradii are different for cold ions and reflected solar wind because of different energies. Therefore, characteristic distances that correspond to the foot region are different for cold ions and reflected solar wind. This can account for the two-layer structure of the foot region. Note that the two-layer structure may accompany two different electric fields. In this case, an ion that entered from the first layer to the second layer experience substantial electric field that accelerates the ion. Therefore, the two-layer structure favors multiple accelerations.

\section{Conclusions}

The foreshock of Venus has similar characteristics to that of the terrestrial foreshock. The VEX/IMA has often observed a combination of field-aligned beams and a gyrating intermediate component traveling away from the Venus bow shock. The observed rather narrow $\delta V_{\|}$cannot be explained by a velocity filter effect of ions leaking from the magnetosheath, while it is consistent with specular reflection of the solar wind. However, the MEX/IMA does not observe such foreshock ions upstream of the Martian bow shock, at least not similar to those observed upstream of Venus using identical instruments. Instead, MEX/IMA has observed a complex zoo of accelerated ions within one proton gyroradius from the quasi-perpendicular bow shock. We further examined a traversal on 12 July 2005, in which large acceleration was ob- served in the magnetic field direction. The accelerated ions close to the bow shock are divided into two categories: collimated ions flowing tailward only within $400 \mathrm{~km}$ of the shock (first layer) and multistep accelerated ions up to $700 \mathrm{~km}$ from the shock (second layer) in addition to pick-up cycloid ions that are observed beyond one proton gyroradius.

The collimated ions in the first layer behave similarly to ions in the foot of the quasi-perpendicular terrestrial bow shock, whereas multistep accelerated ions with energybunching structure are unique to Mars. The velocities of the multistep accelerated ions appear consistent with the multiple reflection of solar wind at the bow shock frank. These ions attain a sufficient field-aligned velocity component for escaping from the bow shock after a second reflection.

While the Mars-Venus difference in the foreshock ion signature in the upstream region of the subsolar bow shock can be attributed to either the curved bow shock or the abundance of cold ions near the Martian bow shock, only the latter may explain the two-layer structure of the shock foot as well as the energy-bunched accelerated ions. To qualitatively test the proposed explanations for the observations, future studies are needed both observationally (i.e., missions with a more complete particle and fields instrument suite) and theoretically. Mars is an ideal space laboratory to study the acceleration process under strong influence of a curved bow shock and the abundance of cold ions in a collisionless plasma.

Acknowledgements. This work was partly supported by the Swedish Research Counsel (VR) \#621-2008-3769 and Swedish National Space Board (RS) in Sweden, and by NASA contract NASW00003 at SwRI in the USA. The lead author (MY) wishes to thank Sweden programs of providing help for physically disabled individuals which have made it possible for him to conduct this work.

Topical Editor I. A. Daglis thanks E. Möbius and another anonymous referee for their help in evaluating this paper.

\section{References}

Alfvén, H. and Fälthammar, C. G.: Cosmical Electrodynamics, Fundamental Principles, Clarendon, Oxford, 1963.

Asbridge, J. R., Bame, S. J., and Strong, I. B.: Outward flow of protons from the Earth's bow shock, J. Geophys. Res., 73, 57775782, 1968.

Barabash S. and Lundin, R.: Reflected Ions Near Mars, Phobos-2 Observations, Geophys. Res. Lett., 20, 787-790, 1993.

Barabash, S., Dubinin, E., Pissarenko, N., Lundin, R., and Russell, C. T.: Picked-up Protons near Mars: Phobos Observations, Geophys. Res. Lett., 18, 1805-1808, 1991.

Barabash, S., Lundin, R., Andersson, H., Brinkfeldt, K., Grigoriev, A., Gunell, H., Holmström, M., Yamauchi, M., Asamura, K., Bochsler, P., Wurz, P., Cerulli-Irelli, R., Mura, A., Milillo, A., Maggi, M., Orsini, S., Coates, A. J., Linder, D. R., Kataria, D. O., Curtis, C. C., Hsieh, K. C., Sandel, B. R., Frahm, R. A., Sharber, J. R., Winningham, J. D., Grande, M., Kallio, E., Koskinen, H., Riihelä, P., Schmidt, W., Säles, T., Kozyra, J. U., Krupp, N., Woch, J., Livi, S., Luhmann, J. G., McKenna-Lawlor, S., Roelof, E. C., Williams, D. J., Sauvaud, J.-A., Fedorov A., 
and Thocaven J.-J.: The analyzer of space plasmas and energetic atoms (ASPERA-3) for the Mars Express mission, Space Sci. Rev., 126(1-4), 113-164, doi:10.1007/s11214-006-9124-8, 2006.

Dubinin, E., Lundin, R., Koskinen, H., and Norberg, O.: Cold Ions at the Martian Bow Shock Phobos Observations, J. Geophys. Res., 98, 5617-5623, 1993.

Dubinin, E. M., Obod, D., Lundin, R., Schwingenschuh, K., and Grard, R.: Some Features of the Martian Bow Shock, Adv. Space Res., 15(8/9), 423-431, 1995.

Dubinin, E. M., Sauer, K., Delva, M., Grard, R., Livi, S., Lundin, R., Skalsky, A., Schwingenschuh, K., Szego, K., and Trotignon, J.-G.: Multi-instrument Study of the Upstream Region near Mars: The Phobos-2 Observations, J. Geophys. Res., 105(A4), 7557-7571, doi:10.1029/1999JA900400, 2000.

Dubinin, E., Fraenz, M., Woch, J., Barabash, S., Lundin, R., and Yamauchi, M.: Hydrogen exosphere at Mars: Pickup protons and their acceleration at the bow shock, Geophys. Res. Lett., 33, L22103, doi:10.1029/2006GL027799, 2006.

Eastwood, J. P., Lucek, E. A., Mazelle, C., Meziane, K., Narita, Y., Pickett, J., and Treumann, R. A.: The foreshock, Space Sci. Rev., 118, 41-94, doi:10.1007/s11214-005-3824-3, 2005.

Eastwood, J. P., Bale, S. D., Mozer, F. S., and Hull, A. J.: Contributions to the cross shock electric field at a quasiperpendicular collisionless shock, Geophys. Res. Lett., 34, L17104, doi:10.1029/2007GL030610, 2007.

Fedorov, A., Budnik, E., Sauvaud, J.-A., Mazelle, C., Barabash, S., Lundin, R., Acuna, M., Holström, M., Grigoriev, A., Yamauchi, M., Andersson, H., Thocaven, J. J., Winningham, D., Frahm, R., Sharber, J. R., Scherrer, J., Coates, A. J., Linder, D. R., Kataria, D. O., Kallio, E., Koskinen, H., Säles, T., Riihelä, P., Schmidt, W., Kozyra, J., Luhmann, J., Roelof, E., Williams, D., Livi, S., Curtis, C. C., Hsieh, K. C., Sandel, B. R., Grande, M., Carter, M., McKenna-Lawler, S., Orsini, S., Cerulli-Irelli, R., Maggi, M., Wurz, P., Bochsler, P., Krupp, N., Woch, J., Fränz, M., Asamura, K., and Dierker, C.: Structure of the Martian wake, Icarus, 182(2), 329-336, doi:10.1016/j.icarus.2005.09.021, 2006.

Fuselier, S. A. and Thomsen, M. F.: He2+ in field aligned beams: ISEE results, Geophys. Res Lett., 19(5), 437-440, 1992.

Fuselier, S. A., Thomsen, M. F., Gosling, J. T., Bame, S. J., and Russell, C. T.: Gyrating and intermediate ion distributions upstream from the Earth's bow shock, J. Geophys. Res., 91(1), 9199, 1986.

Giacalone, J., Jokipii, J. R., and Kóta, J.: Ion injection and acceleration at quasi-perpendicular shocks, J. Geophys. Res., 99, 1935119358, 1994.

Gosling, J. T., Asbridge, J. R., Bame, S. J., Paschmann, G., and Sckopke, N.: Observations of two distinct populations of bow shock ions in the upstream solar wind, Geophys. Res. Lett., 5(11), 957960, 1978.

Gosling, J. T., Thomsen, M. F., Bame, S. J., Feldman, W. C., Paschmann, G., and Sckopke, N.: Evidence for specularly reflected ions upstream from the quasi-parallel bow shock, Geophys. Res. Lett., 9(12), 1333-1336, 1982.

Grard, R., Nairn, C., Pedersen, A., Klimov, S., Savin, S., Skalsky, A., and Trotignon, J. G.: Plasma and Waves around Mars, Planet. Space Sci., 39, 89-98, 1991.

Holmström, M., Wieser, M., Barabash, S., Futaana, Y., and Bhardwaj, A.: Dynamics of solar wind protons reflected by the Moon,
J. Geophys. Res., 115, A06206, doi:10.1029/2009JA014843, 2010.

Klassen, A., Gómez-Herrero, R., Müller-Mellin, R., Böttcher, S., Heber, B., Wimmer-Schweingruber, R., and Mason, G. M.: STEREO/SEPT observations of upstream particle events: almost monoenergetic ion beams, Ann. Geophys., 27, 2077-2085, doi:10.5194/angeo-27-2077-2009, 2009.

Kucharek, H., Möbius, E., Scholer, M., Mouikis, C., Kistler, L. M., Horbury, T., Balogh, A., Réme, H., and Bosqued, J. M.: On the origin of field-aligned beams at the quasi-perpendicular bow shock: multi-spacecraft observations by Cluster, Ann. Geophys., 22, 2301-2308, doi:10.5194/angeo-22-2301-2004, 2004.

Lee, M. A., Shapiro, V. D., and Sagdeev, R. Z.: Pickup ion energization by shock surfing, J. Geophys. Res., 101(A3), 4777-4789, 1996.

Lutsenko, V. N. and Kudela, K.: Almost monoenergetic ions near the Earth's magnetosphere boundaries, Geophys. Res. Lett., 26, 413-416, 1999.

Mazelle, C., Meziane, K., LeQuéau, D., Wilber, M., Eastwood, J. P., Rème, H., Sauvaud, J.-A., Bosqued,J. M., Dandouras, I., McCarthy, M., Kistler, L. M., Klecker, B., Korth, A., BavassanoCattaneo, M. B., Pallocchia, G., Lundin, R., and Balogh, A: Production of gyrating ions from nonlinear wave-particle interaction upstream from the Earth's bow shock: a case study from ClusterCIS, Planet. Space Sci., 51, 785-795, 2003.

Mazelle, C., Winterhalter, D., Sauer, K., Trotignon, J. G., Acuna, M. H., Baumgärtel, K., Bertucci, C., Brain, D. A., Brecht, S. H., Delva, M., Dubinin, E., Øieroset, M., and Slavin, J.: Bow shock and upstream phenomena at Mars, Space Sci. Rev., 111, 115-181, 2004.

Meziane, K., Mazelle, C., Wilber, M., LeQuéau, D., Eastwood, J. P., Rème, H., Dandouras, I., Sauvaud, J. A., Bosqued, J. M., Parks, G. K., Kistler, L. M., McCarthy, M., Klecker, B., Korth, A., Bavassano-Cattaneo, M.-B., Lundin, R., and Balogh, A.: Bow shock specularly reflected ions in the presence of low-frequency electromagnetic waves: a case study, Ann. Geophys., 22, 23252335, doi:10.5194/angeo-22-2325-2004, 2004a.

Meziane, K., Wilber, M., Mazelle, C., LeQuéau, D., Kucharek, H., Lucek, E.A., Rème, H., Hamza, A. M., Sauvaud, J.-A., Bosqued, J. M., Dandouras, I., Parks, G. K., McCarthy, M., Klecker, B., Korth, A., Bavassano-Cattaneo, M. B., and Lundin, R. N.: Simultaneous observations of field-aligned beams and gyrating ions in the terrestrial foreshock, J. Geophys. Res., 109, A05107, doi:10.1029/2003JA010374, 2004b.

Miao, B., Kucharek, H., Möbius, E., Mouikis, C., Matsui, H., Liu, Y. C.-M., and Lucek, E. A.: Remote sensing of local structure of the quasi-perpendicular Earth's bow shock by using field-aligned beams, Ann. Geophys., 27, 913-921, doi:10.5194/angeo-27913-2009, 2009.

Möbius, E., Kucharek, H., Mouikis, C., Georgescu, E., Kistler, L. M., Popecki, M. A., Scholer, M., Bosqued, J. M., Rème, H., Carlson, C. W., Klecker, B., Korth, A., Parks, G. K., Sauvaud, J. C., Balsiger, H., Bavassano-Cattaneo, M.-B., Dandouras, I., DiLellis, A. M., Eliasson, L., Formisano, V., Horbury, T., Lennartsson, W., Lundin, R., McCarthy, M., McFadden, J. P., and Paschmann, G.: Observations of the spatial and temporal structure of field-aligned beam and gyrating ring distributions at the quasi-perpendicular bow shock with Cluster CIS, Ann. Geophys., 19, 1411-1420, doi:10.5194/angeo-19-1411-2001, 2001. 
Nishino, M. N., Fujimoto, M., Maezawa, K., Saito, Y., Yokota, S., Asamura, K., Tanaka, T., Tsunakawa, H., Matsushima, M., Takahashi, F., Terasawa, T., Shibuya, H., and Shimizu, H.: Solarwind proton access deep into the near-Moon wake, Geophys. Res. Lett., 36, L16103, doi:10.1029/2009GL039444, 2009.

Oka, M., Terasawa, T., Saito, Y., and Mukai, T.: Field-aligned beam observations at the quasi-perpendicular bow shock: Generation and shock angle dependence, J. Geophys. Res., 110, A05101, doi:10.1029/2004JA010688, 2005.

Paschmann, G., Sckopke, N., Asbridge, J. R., Bame, S. J., and Gosling, J. T., Energization of solar wind ions by reflection from the Earth's bow shock, J. Geophys. Res., 85, 4689-4693, 1980.

Paschmann, G., Sckopke, N., Papamastorakis, I., Asbridge, J. R., Bame, S. J., and Gosling, J. T.: Characteristics of reflected and diffuse ions upstream from the Earth's bow shock, J. Geophys. Res. 86, 4355-4364, 1981.

Sagdeev, R. Z.: Cooperative phenomena and shock waves in collision-less plasmas, in: Reviews of Plasma Physics, vol. 4, edited by: Leontovich, M. A., p. 23, Consultants Bur., New York, 1966.

Sarris, E. T., Anagnostopoulos, G. C., and Krimigis, S. M.: Simultaneous measurements of energetic ion $(50 \mathrm{keV}$ and above $)$ and electron (220 keV and above) activity upstream of Earth's bow shock and inside the plasma sheet - Magnetospheric source for the November 3 and December 3, 1977 upstream events, J. Geophys. Res., 92(11), 12083-12096, 1987.

Sckopke, N., Paschmann, G., Bame, S. J., Gosling, J. T., and Russell, C. T., Evolution of ion distributions across the nearly perpendicular bow shock: Specularly and non-specularly reflectedgyrating ions, J. Geophys. Res., 88, 6121-6136, 1983.

Sckopke, N., Paschmann, G., Brinca, A. L., Carlson, C. W., and Luhr, H.: Ion thermalization in quasi-perpendicular shocks involving reflected ions, J. Geophys. Res., 95, 6337-6352, 1990.

Sibeck, D. G., Omidi, N., Dandouras, I., and Lucek, E.: On the edge of the foreshock: model-data comparisons, Ann. Geophys., 26, 1539-1544, doi:10.5194/angeo-26-1539-2008, 2008.

Slavin, J. A., Holzer, R. E., Spreiter, J. R., and Stahara, S. S., Planetary Mach Cones: Theory and Observation, J. Geophys. Res., 89(A5), 2708-2714, 1984.
Smith, E. J. and Wolfe, J. H.: Fields and plasmas in the outer solar system, Space Sci. Rev., 23(2), 217-252, 1979.

Tanaka, M., Goodrich, C. C., Winske, D., and Papadopoulos, K.: A source of the backstreaming ion beams in the foreshock region, J. Geophys. Res., 88, 3046-3054, 1983.

Winningham, J. D., Frahm, R. A., Sharber, J. R., Coates, A. J., Linder, D. R., Soobiah, Y., Kallio, E., Espley, J. R., Lundin, R., Barabash, S., Holmström, M., Andersson, H., Yamauchi, M., Grigoriev, A., Scherrer, J. R., Jeffers, S. J., Kataria, D. O., Kozyra, J. U., Luhmann, J. G., Roelof, E. C., Williams, D. J., Livi, S., Curtis, C. C., Hsieh, K. C., Sandel, B. R., Koskinen, H., Säles, T., Riihelä, P., Schmidt, W., Grande, M., Carter, M., Sauvaud, J.-A., Fedorov, A., Thocaven, J.J., McKenna-Lawler, S., Orsini, S., Cerulli-Irelli, R., Maggi, M., Wurz, P., Bochsler, P., Krupp, N., Woch, J., Fränz, M., Asamura, K., and Dierker, C.: Electron oscillations in the induced Martian magnetosphere, Icarus, 182(2), 360-370, doi:10.1016/j.icarus.2005.10.033, 2006.

Yamauchi, M., Futaana, Y., Fedorov, A., Dubinin, E., Lundin, R., Sauvaud, J.-A., Winningham, D., Frahm, R., Barabash, S., Holmström, M., Woch, J., Fraenz, M., Budnik, E., Borg, H., Sharber, J. R., Coates, A. J., Soobiah, Y., Koskinen, H., Kallio, E., Asamura, K., Hayakawa, H., Curtis, C., Hsieh, K. C., Sandel, B. R., Grande, M., Grigoriev, A., Wurz, P., Orsini, S., Brandt, P., McKenna-Lawler, S., Kozyra, J., and Luhmann, J.: IMF direction derivation from cycloid-like ion distributions observed by Mars Express, Space Sci. Rev., 126(1-4), 239-266, doi:10.1007/s11214-006-9090-1, 2006.

Yamauchi, M., Futaana, Y., Fedorov, A., Kallio, E., Frahm, R. A., Lundin, R., Sauvaud, J.-A., Winningham, D. J., Barabash, S., and Holmström, M.: Advanced method to derive the IMF direction near Mars from cycloidal proton distributions, Planet. Space Sci., 56(8), 1145-1154, doi:10.1016/j.pss.2008.02.012, 2008.

Zank, G. P., Pauls, H. L., Cairns, I. H., and Webb, G. M.: Interstellar pickup ions and quasi-perpendicular shocks: Implications for the termination shock and interplanetary shocks, J. Geophys. Res., 101(A1), 457-477, 1996. 